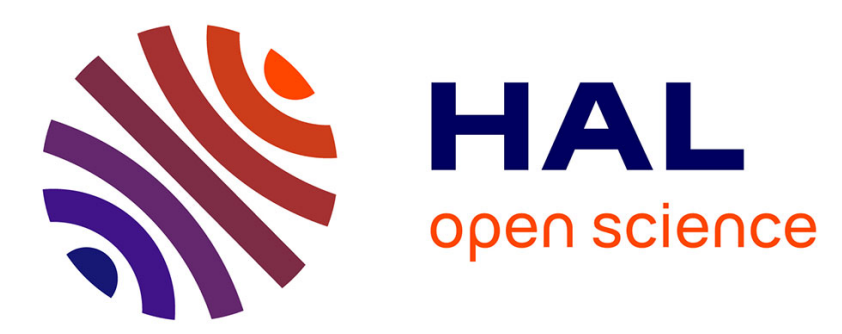

\title{
Out-of-plane deformation reduction via inelastic hinges in fibrous metamaterials and simplified damage approach
}

Mario Spagnuolo, M Erden Yildizdag, Xavier Pinelli, Antonio Cazzani, François Hild

\section{- To cite this version:}

Mario Spagnuolo, M Erden Yildizdag, Xavier Pinelli, Antonio Cazzani, François Hild. Out-of-plane deformation reduction via inelastic hinges in fibrous metamaterials and simplified damage approach. Mathematics and Mechanics of Solids, 2022, 27 (6), pp.1011-1031. 10.1177/10812865211052670 . hal-03494141

\author{
HAL Id: hal-03494141 \\ https://hal.science/hal-03494141
}

Submitted on 18 Dec 2021

HAL is a multi-disciplinary open access archive for the deposit and dissemination of scientific research documents, whether they are published or not. The documents may come from teaching and research institutions in France or abroad, or from public or private research centers.
L'archive ouverte pluridisciplinaire HAL, est destinée au dépôt et à la diffusion de documents scientifiques de niveau recherche, publiés ou non, émanant des établissements d'enseignement et de recherche français ou étrangers, des laboratoires publics ou privés. 


\title{
Out-of-plane deformation reduction via inelastic hinges in fibrous metamaterials and simplified damage approach
}

Journal Title

$\mathrm{XX}(\mathrm{X}): ? ?-?$ ?

(C)The Author(s) 2016

Reprints and permission:

sagepub.co.uk/journalsPermissions.nav DOI: $10.1177 /$ ToBeAssigned www.sagepub.com/

\section{SAGE}

\author{
Mario Spagnuolo ${ }^{1,2}$, M. Erden Yildizdag ${ }^{2,3}$, Xavier Pinelli ${ }^{4}$, Antonio \\ Cazzani ${ }^{1}$, François Hild ${ }^{4}$
}

\begin{abstract}
The mechanical behavior of fibrous metamaterials is mainly determined by the interactions between the fibers composing the architecture. These interactions are usually of two different kinds: the ones directly depending on the positions of the fibers and those that need mediators, usually consisting in hinges, either inelastic or perfect, inducing restrictions on the kinematics of the fiber joints. In cases of interest, it has been observed that hinges can either have a certain torsional stiffness or behave as perfect joints, simply ensuring that the fibers remain interconnected, but not applying any constraint on the relative rotations between them. Here the effect of torsional stiffness of inelastic hinges is studied in two shear tests for a selected fibrous metamaterial. It is shown that the stiffness of hinges can be tailored to avoid, or at least reduce, out-of-plane deformations. Moreover, it is shown that, after reaching a threshold, permanent deformations are observed. This phenomenon is treated in a simplified way, by introducing damage in the continuum model.
\end{abstract}

\section{Keywords}

Fibrous metamaterials, Higher gradient theories, Buckling, Digital Image Correlation, Damage 


\section{Introduction}

In the classification of mechanical metamaterials, a very sophisticated and varied class is represented by fibrous metamaterials, i.e. metamaterials whose microstructure (or architecture, where the term micro refers to relationship between two scales of observation, the global or macro scale and that of the architecture or micro scale) is composed of assemblages of fibers.

These metamaterials are of interest for at least two reasons: (i) the simplicity of production and (ii) their high performance mechanical properties. First, it is noted that in nature there are materials whose mechanical properties are determined by the intrinsic microstructure. An interesting aspect is that such natural microstructures are often composed of differently arranged fibers. Different morphologies generate significantly different mechanical properties at the macroscopic level. This is a fundamental aspect underlying the search for new artificial microstructures.

Remarkable examples of naturally occurring fibrous microstructures can be found in bone tissues or in wood??? ? ? ? ? ? The fact that many natural microstructures have fibrous components empirically demonstrates how the production of these microstructures can be extremely easy and, above all, extremely adaptive. The case of bone tissues epitomizes how microstructures are obtained through topological optimization thus ensuring that the result, once the objective function (which, from a practical point of view, corresponds to the mechanical property) has been chosen, is the best possible? ? .

The criterion of simplicity of production is certainly true for metamaterials (or architectured materials) consisting of fibrous microstructures. The possibilities opened by the rapid development of additive manufacturing technologies are huge ? ?

In contrast to what is proposed by the so-called avant-garde schools (see for example ${ }^{\text {, }}$, where the macro behavior of the metamaterial is studied a posteriori and microstructures are "randomly" chosen hoping that exotic mechanical properties arise), the present approach starts from a priori chosen mechanical properties and, from those, searches for microstructures that allow, on the basis of selected homogenization

\footnotetext{
${ }^{1}$ Dipartimento di Ingegneria Civile, Ambientale e Architettura (DICAAR), Università degli Studi di Cagliari, Cagliari, Italy

${ }^{2}$ International Research Center for the Mathematics and Mechanics of Complex Systems, University of L'Aquila, Italy

${ }^{3}$ Faculty of Naval Architecture and Ocean Engineering, Istanbul Technical University, 34469, Maslak, Istanbul, Turkey

${ }^{4}$ Université Paris-Saclay, ENS Paris-Saclay, CNRS; LMT - Laboratoire de Mécanique et Technologie, Gifsur-Yvette, France
}

\section{Corresponding author:}

M. Erden Yildizdag

Email: yildizdag@itu.edu.tr 
techniques, to associate the required macro property with the sought microstructural one ? ? ? ? ? . From this point of view, metamaterials can be considered as materials on demand? ? ? ? ? ? ? . This work focuses on some properties of fibrous metamaterials.

The mechanical properties of fibrous metamaterials are mainly influenced by the interactions between the fibers that constitute the microstructure. These interactions are either direct or indirect. On the one side, direct interactions primarily depend on

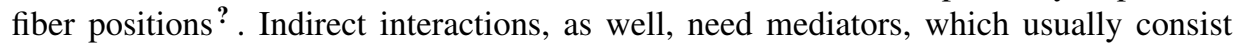
of hinges (or pivots), either inelastic or perfect, which restrict the kinematics of the fiber joints?????? . In this study the role of hinges is studied in the response

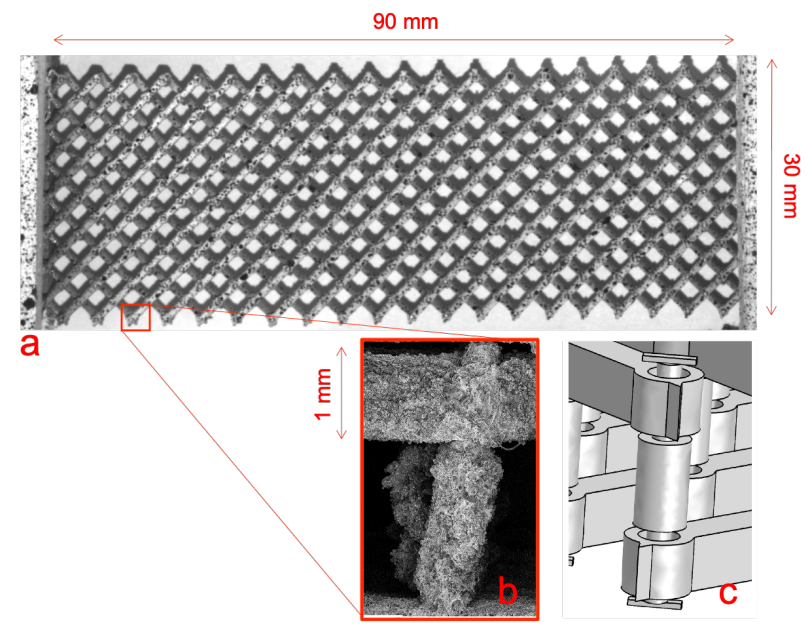

Figure 1. Example of 3D printed pantographic structure with quasi-perfect pivots. (a) Full specimen. (b) SEM image of a single pivot. (c) Corresponding CAD design.

of fibrous metamaterials. One of the simplest cases is represented by pantographic metamaterials????????? This metamaterial is composed of mutually orthogonal families of fibers that are held together by cylindrical joints, which in the literature (see Fig. ??) are generally called pivots. These structural elements can either have a certain torsional stiffness, and thus add an additional deformation mechanism to that of the metamaterial, or behave as perfect hinges, ensuring only that the two fiber families remain interconnected, but not exerting any constraint on the relative rotations between the fibers. Between these two states of constraint and absence of thereof, there is a whole class of possible hinge stiffnesses. If the above-mentioned torsional stiffness approaches zero but is yet significantly different from such value, then such elements are referred to as inelastic hinges or quasi-perfect pivots? . The importance of these hinges lies in the fact that in the macroscopic homogenised response they are related to shear contributions to the strain energy. Quasi-perfect pivots have the property of considerably decreasing, if not eliminating at all, the shear energy contribution? 
This aspect is perhaps the most immediately visible, but quasi-perfect pivots exhibit a second important feature. They offer a higher degree of stability to the structure than that observed with standard pivots. The experimental analyses reported herein address this point. Two specimens were subjected to shear. The main difference between them was that one had standard pivots while the other had quasi-perfect pivots. The overall dimensions of the objects were the same, but the number of pivots and fibers did not coincide in both cases. Even though one may think that it is not possible to make a proper comparison between the two specimens, it has been recently observed that this type of metamaterial can be correctly described through continuum homogenized models even if its microstructure is not very dense? ${ }^{\text {? }}$. This statement implies that, assuming the same printing material and global dimensions, the comparison between specimens with microstructures with different spacings is not unreliable and, on the contrary, is made possible when considering the continuum description.

A remarkable result that emerges from the comparison between the two shear tests is the fact that out-of-plane deformations are observed for standard hinges with fixed torsional rigidity and is almost absent in the case of quasi-perfect-pivots. This phenomenon is reproducible through numerical simulations based on a 3D model of the pantographic metamaterial ${ }^{\text {? }}$. The interpretation of the main difference between the two cases lies in a problem of stability.

\section{Second gradient homogenized model}

It has been shown? ? ? that, if one wants to approach Continuum Mechanics by taking into account the presence of microstructures, then in some particular cases it is necessary to introduce in the strain energy terms depending on the second gradient of displacement. Even when the micro and macro scales are not decisively separated, the continuum second gradient model can be used to obtain estimates that are very close to experiments? .

The second gradient model ? ? is a two-dimensional description, suitable for the characterization of tests that induce strains and stresses only in the plane. It is characterized by a strain energy written in terms of the placement field and its derivatives, which associates each point of the reference configuration to its position in the deformed configuration (see Fig. ?? for the adopted nomenclature). Let $\left(\mathbf{D}_{1}, \mathbf{D}_{2}\right)$ be an orthogonal basis for the reference configuration. For simplicity, $\left(\mathbf{D}_{1}, \mathbf{D}_{2}\right)$ are taken along the directions of the two fiber families which are, in the reference configuration, orthogonal to each other. Let us now consider a $2 \mathrm{D}$ continuum whose reference shape is given by a rectangular domain $\Omega=\left[0, \mathrm{~L}_{a}\right] \times\left[0, \mathrm{~L}_{b}\right] \subset \mathbb{R}^{2}$, where $\mathrm{L}_{a}$ and $\mathrm{L}_{b}$ are the lengths of the sides of the pantographic structure (Fig. ??). Very often, it is assumed that $\mathrm{L}_{a}=3 \mathrm{~L}_{b}$. If only planar motions are considered, then the current shape of the rectangle is mathematically described by a regular placement function $\chi: \Omega \rightarrow \mathbb{R}^{2}$. By employing the so-called Piola's Ansatz and assuming that $\chi(\cdot)$ is at least twice differentiable, the 


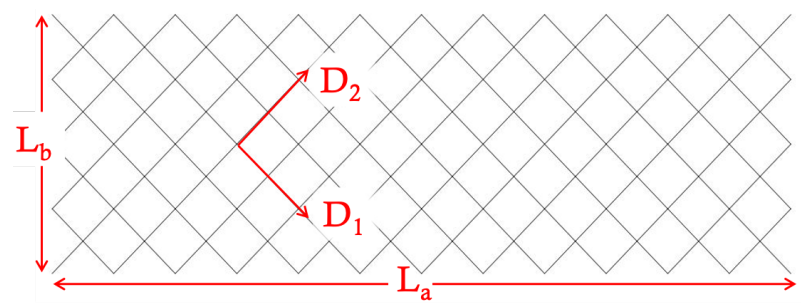

Figure 2. Reference configuration of a pantographic structure.

macroscopic strain energy becomes for the pantographic metamaterial ${ }^{?}$

$$
\begin{aligned}
\mathcal{U}(\chi(\cdot)) & =\int_{\Omega} \sum_{\alpha} \frac{\mathrm{K}_{e}^{\alpha}}{2}\left(\left\|\boldsymbol{F} \mathbf{D}_{\alpha}\right\|-\mathbf{1}\right)^{\mathbf{2}} \mathbf{d} \boldsymbol{\Omega} \\
& +\int_{\Omega} \sum_{\alpha} \frac{\mathrm{K}_{b}^{\alpha}}{2}\left[\frac{\nabla \boldsymbol{F}\left|\mathbf{D}_{\alpha} \otimes \mathbf{D}_{\alpha} \cdot \nabla \boldsymbol{F}\right| \mathbf{D}_{\alpha} \otimes \mathbf{D}_{\alpha}}{\left\|\boldsymbol{F} \mathbf{D}_{\alpha}\right\|^{\mathbf{2}}}\right. \\
& \left.-\left(\frac{\boldsymbol{F} \mathbf{D}_{\alpha}}{\left\|\boldsymbol{F} \mathbf{D}_{\alpha}\right\|} \cdot \frac{\nabla \boldsymbol{F} \mid \mathbf{D}_{\alpha} \otimes \mathbf{D}_{\alpha}}{\left\|\boldsymbol{F} \mathbf{D}_{\alpha}\right\|}\right)^{2}\right] d \Omega
\end{aligned}
$$

where $\boldsymbol{F}$ denotes the deformation gradient $\nabla \boldsymbol{\chi}$, and $\left(\nabla \boldsymbol{F} \mid \mathbf{D}_{\alpha} \otimes \mathbf{D}_{\alpha}\right)^{\beta}=F_{\alpha, \alpha}^{\beta}=\chi_{, \alpha \alpha}^{\beta}$. Note that Greek indices assume values in the range 1,2. The first integral in Eq. (??) accounts for the fiber elongation energy ( $\mathrm{K}_{e}$ is the elongation stiffness) while the second integral represents the fiber bending energy ( $K_{b}$ being the elongation stiffness). It is worth noting that the bending energy is written in terms of the gradient of the deformation tensor $\nabla \boldsymbol{F}$ corresponding to a second gradient of the placement field $\nabla^{2} \chi$. In the strain energy (??) no energetic term related to pivots was added. This is the case when pivots are perfect.

When out-of-plane phenomena need to be considered, the model must be extended. The pantographic layer is first viewed as a network of beams, where the motion of each beam is described by a placement function of the beam axis (i.e. line) and a rotation

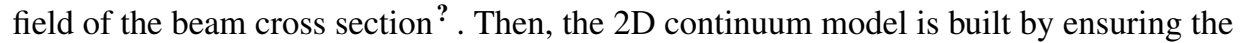
equivalence with the network model through the strain energy density.

In the continuum model, see Fig. ??, as the two families of fibers are orthogonally aligned in the reference configuration $\mathcal{R}$, a cartesian basis $\left\{\mathbf{D}_{1}, \mathbf{D}_{2}, \mathbf{D}_{3}\right\}$ is introduced such that $\mathbf{D}_{1}$ and $\mathbf{D}_{2}$ are chosen to be directed along the unit tangent vectors of each family of fibers (i.e. $\mathbf{D}_{1}=\mathbf{E}_{1}$ and $\mathbf{D}_{2}=\mathbf{E}_{2}$ ). Accordingly, the unit vectors $\mathbf{M}_{\alpha}=$ $\mathbf{N} \times \mathbf{E}_{\alpha}$ and $\mathbf{N}=\mathbf{E}_{1} \times \mathbf{E}_{2}$ are introduced along the principal inertial axes of the beam cross sections $\left(\mathbf{D}_{3}=\mathbf{N}\right)$. Therefore, any material point $X \in \mathcal{R}$ has the coordinates $\left(X_{1}, X_{2}\right)$ in the reference configuration. 


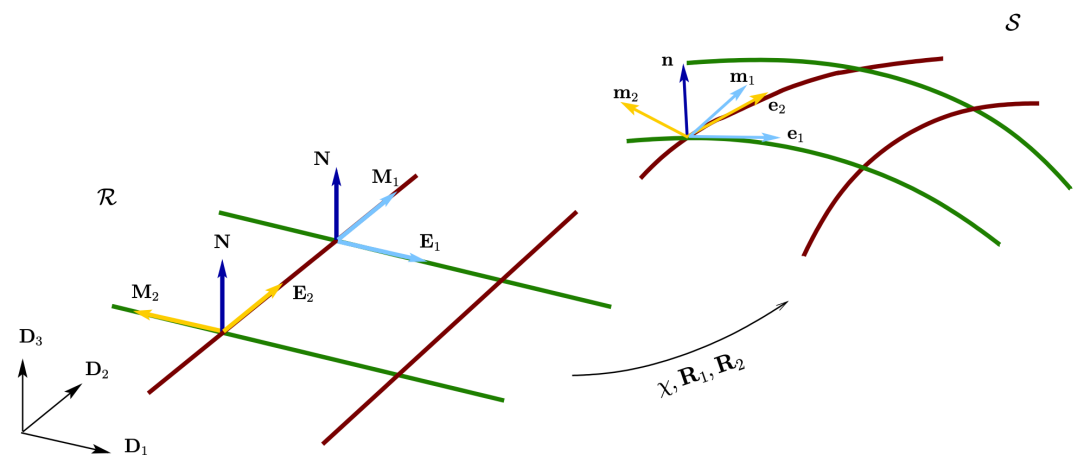

Figure 3. 2D continuum with microstructure in the reference $\mathcal{R}$ and deformed $\mathcal{S}$ configurations

The motion of pantographic structures is described by placement $\chi: \mathcal{R} \rightarrow \mathcal{S}$ and two orthogonal tensor fields give the orientation of the deformed microstructure

$$
\begin{aligned}
& \mathbf{R}_{1}:\left(\mathbf{D}_{1}, \mathbf{D}_{2}, \mathbf{D}_{3}\right) \rightarrow \mathbf{e}_{1}, \mathbf{m}_{1}, \mathbf{n} \\
& \mathbf{R}_{2}:\left(\mathbf{D}_{1}, \mathbf{D}_{2}, \mathbf{D}_{3}\right) \rightarrow \mathbf{e}_{2}, \mathbf{m}_{2}, \mathbf{n} .
\end{aligned}
$$

where $\mathbf{n}$ is the unit normal vector

$$
\mathbf{n}=\frac{\mathbf{e}_{1} \times \mathbf{e}_{2}}{\left\|\mathbf{e}_{1} \times \mathbf{e}_{2}\right\|}
$$

and $\mathbf{e}_{\alpha}$ denotes the unit tangent vectors

$$
\mathbf{e}_{\alpha}=\frac{\partial \chi / \partial \mathbf{X}_{\alpha}}{\left\|\partial \chi / \partial \mathbf{X}_{\alpha}\right\|}
$$

in the reference configuration. More details on the nature of the orthogonal tensors $\mathbf{R}_{\alpha}$ are given in?. Then, the displacement vector may be expressed as follows

$$
\mathbf{u}(\mathbf{X})=\mathbf{x}-\mathbf{X}=u_{1}(\mathbf{X}) \mathbf{D}_{1}+u_{2}(\mathbf{X}) \mathbf{D}_{2}+u_{3}(\mathbf{X}) \mathbf{D}_{3}
$$

In the model, the strain energy density

$$
W=W_{e}+W_{s}+W_{t}+W_{n}+W_{g}
$$

is introduced, where $W_{e}, W_{s}, W_{t}, W_{n}$, and $W_{g}$ are the terms related to elongation, shear, twist, normal bending, and geodesic bending respectively. The last three terms are related to the three possible flexural deformations, occurring along the principal directions of the fiber cross-section. For more details about this formulation and the need of introducing a local reference (the so-called moving trihedron) in case of curved surfaces, the reader is 
referred to the interesting book ${ }^{?}$. In the present work the same notation as in ${ }^{?}$ is adopted.

$$
\begin{aligned}
W_{e} & =\frac{1}{2} K_{e}\left(\varepsilon_{1}^{2}+\varepsilon_{2}^{2}\right), \\
W_{s} & =\frac{1}{2} K_{s h} \gamma^{2}, \\
W_{t} & =\frac{1}{2} K_{t}\left(\kappa_{11}^{2}+\kappa_{12}^{2}\right), \\
W_{n} & =\frac{1}{2} K_{b n}\left(\kappa_{21}^{2}+\kappa_{22}^{2}\right), \\
W_{g} & =\frac{1}{2} K_{b g}\left(\kappa_{31}^{2}+\kappa_{32}^{2}\right) .
\end{aligned}
$$

where $\varepsilon$ and $\gamma$ are the local strain measures related to stretching

$$
\varepsilon=\left\|\mathbf{t}_{\alpha}\right\|-1, \quad \gamma=\sin ^{-1}\left(\mathbf{e}_{1} \cdot \mathbf{e}_{2}\right)
$$

with

$$
\mathbf{t}_{\alpha}=\frac{\partial \chi}{\partial \mathbf{X}_{\alpha}}
$$

the terms $\kappa_{1 \alpha}, \kappa_{2 \alpha}$, and $\kappa_{3 \alpha}$ are the geodesic torsion, normal curvature, and geodesic curvature

$$
\begin{aligned}
\kappa_{11} & =-\frac{\mathbf{n} \cdot \mathbf{g}_{1}-\sin \gamma \mathbf{n} \cdot \mathbf{c}_{1}}{\left\|\mathbf{e}_{1} \times \mathbf{e}_{2}\right\|}, & \kappa_{12} & =\frac{\mathbf{n} \cdot \mathbf{g}_{2}-\sin \gamma \mathbf{n} \cdot \mathbf{c}_{2}}{\left\|\mathbf{e}_{1} \times \mathbf{e}_{2}\right\|} \\
\kappa_{21} & =\mathbf{n} \cdot \mathbf{c}_{1}, & \kappa_{22} & =\mathbf{n} \cdot \mathbf{c}_{2} \\
\kappa_{31} & =-\mathbf{m}_{1} \cdot \mathbf{c}_{1}, & \kappa_{32} & =-\mathbf{m}_{2} \cdot \mathbf{c}_{2}
\end{aligned}
$$

and

$$
\begin{array}{ll}
\mathbf{c}_{1}=\frac{\nabla \nabla \chi\left(\mathbf{D}_{1} \otimes \mathbf{D}_{1}\right)}{\left\|\nabla \chi \mathbf{D}_{1}\right\|}, & \mathbf{c}_{2}=\frac{\nabla \nabla \chi\left(\mathbf{D}_{2} \otimes \mathbf{D}_{2}\right)}{\left\|\nabla \chi \mathbf{D}_{2}\right\|}, \\
\mathbf{g}_{1}=\frac{\nabla \nabla \chi\left(\mathbf{D}_{2} \otimes \mathbf{D}_{1}\right)}{\left\|\nabla \chi \mathbf{D}_{2}\right\|}, & \mathbf{g}_{2}=\frac{\nabla \nabla \chi\left(\mathbf{D}_{1} \otimes \mathbf{D}_{2}\right)}{\left\|\nabla \chi \mathbf{D}_{1}\right\|} .
\end{array}
$$

In addition, the terms $K_{e}, K_{s}, K_{t}, K_{n}$, and $K_{g}$ are the associated stiffnesses

$$
K_{e}=\frac{E A}{\ell}, \quad K_{s h}=\frac{G J_{p}}{h_{p} \ell^{2}}, \quad K_{t}=\frac{G J_{t}}{\ell}, K_{b n}=\frac{E J_{f_{1}}}{\ell}, \quad K_{b g}=\frac{E J_{f_{2}}}{\ell} .
$$

where $E$ is the Young's modulus, $G$ the shear modulus, $J_{p}$ torsional inertia of the hinge, $J_{t}, J_{f_{1}}, J_{f_{2}}$ the torsional and flexural inertias of the beam cross section? ? ? . Further, $\ell$ is the distance between two adjacent pivots, $h_{p}$ the height of the pivot and $A$ the cross sectional area of the beam. The governing equations are obtained with the variational 
statement

$$
\delta \int_{\Omega} W\left({ }^{\alpha} \varepsilon, \gamma,{ }^{\alpha} \kappa_{1},{ }^{\alpha} \kappa_{2},{ }^{\alpha} \kappa_{3}\right) d \Omega=0 \quad \forall \delta \mathbf{u}
$$

where $\delta \mathbf{u}$ belongs to the vector space of admissible displacement variations. For further details on the continuum model, the reader is referred to?

\section{Stability and kinematics}

While in bias extension tests it is less common to observe out-of-plane deformations, in shear tests this type of deformation may occur more easily. One of the possible approaches to the study of this type of phenomenon is based on the analysis of stability. In the case of pantographic structures, the degrees of stability are strongly related to the deformation mode of the various elements. The study presented herein aims at observing the emergence of these phenomena in structures with the same kind of microstructural elements, except the hinges which interconnect the two families of fibers.

Equilibrium is obtained at particular configurations where the sum of the various energy terms introduced for the pantographic structure gives rise to a minimum energy. In this context the stiffnesses associated with each of the energy terms play a fundamental role. In fact, depending on the set of stiffnesses that are considered, one deformation mechanism will be favored over another since, for the same prescribed displacement, it will result in an energy minimum:

i. the first mechanisms to be activated are fiber bending and hinge torsion;

ii. the bending energy of the fibers is negligible compared to that associated with torsion of hinges;

iii. when the torsional stiffness of hinges is large enough, a minimum of the total energy is found in out-of-plane configurations.

As it is displayed in Fig. ??, an out-of-plane deformation is observed in the case of standard hinges, which are not present in the case when the torsional stiffness is lower.

\section{Simplified approach to pivot inelasticity}

In Fig. ??, the reaction force versus displacement is presented for the two specimens. The main difference is in the order of magnitude of the maximum force the samples are subjected to. This difference is due to the fact that in one of the two specimens the hinges are almost perfect and, consequently, the force is much lower than in the case of so-called standard pivots. From a qualitative point of view, similar trends are observed in both cases. The curve starts with a certain slope. Then, for a certain displacement step, $u^{*}$, the slope decreases considerably. Last, the slope increases again, this time almost asymptotically, just before the first observed break. The first change in slope is not exactly due to the same mechanism in both cases. For the standard case, plasticity is occurring in hinges while in the other case the mechanism is believed to be more 


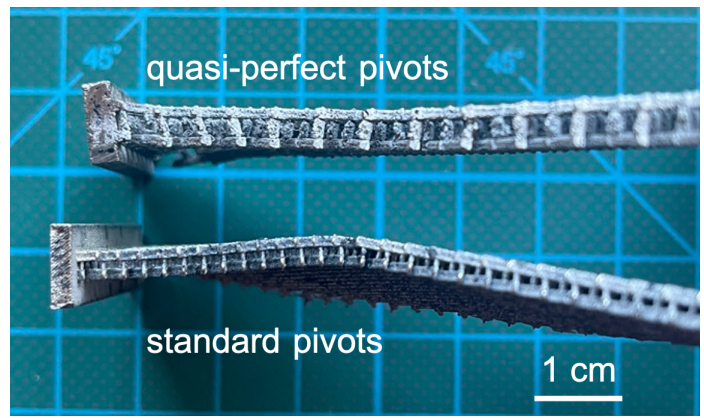

Figure 4. Out-of-plane deformation of the specimen with standard pivots. Comparison with the quasi-perfect pivots case after failure due to shear tests.

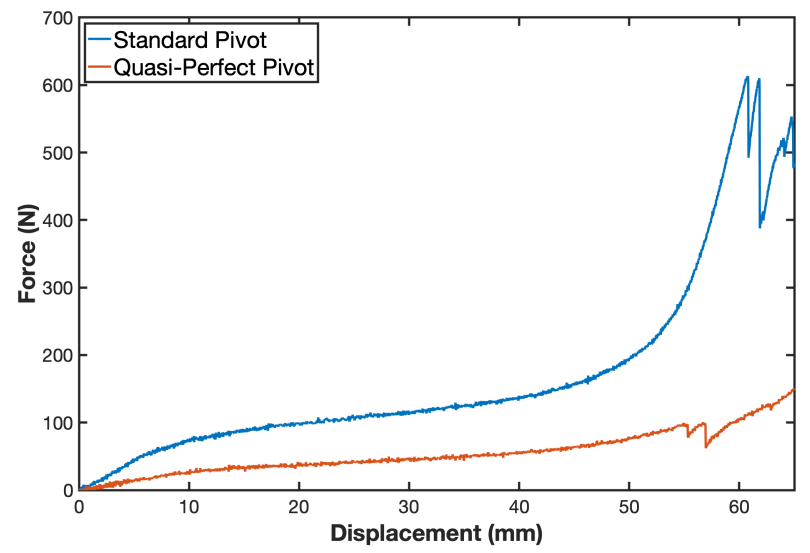

Figure 5. Measured reaction force versus prescribed displacement for standard pivots (blue) and quasi-perfect pivots (red) specimens.

subtle. Simultaneously to yielding, fiber bending is also activated, which is modeled by the second gradient term. However, the energy associated with fiber deformation is much lower than that associated with torsion of cylinders of the same material.

When the angle between the fibers of the two families approaches zero, i.e. when the fibers are parallel, then the prevalent deformation mechanism becomes the elongation of the fibers. The activation of the deformation mechanism by fiber extension corresponds to the second change in slope observed in Fig. ??. Since this paper focuses on hinges as structural elements, the discussion is limited to the first change of slope.

From a phenomenological point of view, inelasticity occurs when the material is stressed beyond its yield point. Even when the load is removed, inelastic (permanent) strains remain (see Fig. ??, where, after breaking, the specimen, which is no longer 
stressed, remains deformed). The activation in the material of permanent strains at the macroscopic level is commonly called yielding. In the case described in Fig. ??, the displacement associated with the yield point corresponds to $u^{*}$.

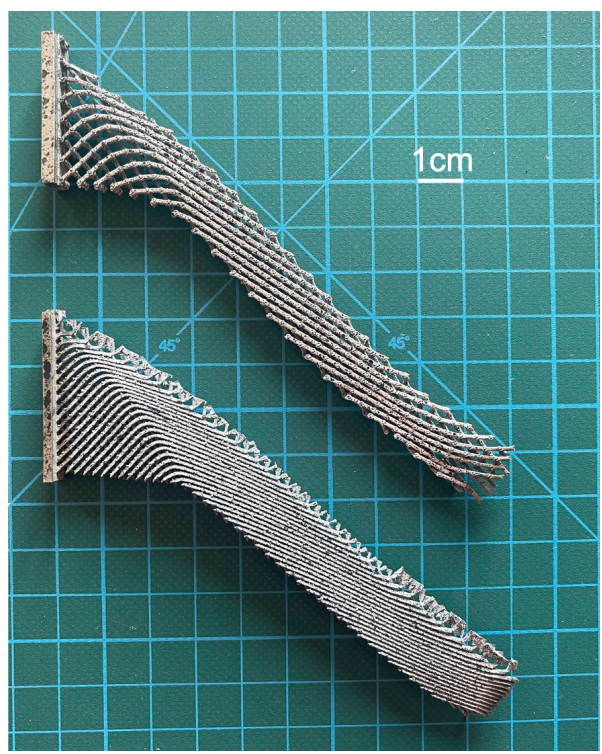

Figure 6. Permanent deformation in the two broken specimens.

Pivot permanent deformation is modeled in the simplest way possible, in order to obtain numerical simulations qualitatively agreeing with the experimental data. A simplified approach considers two aspects among those reported above:

i. the first part of the deformation process of the pantographic structures is characterized by torsional deformation of the pivots from an energetic point of view, so the initial slope is strongly related to the torsional stiffness of the pivots (which, from a macroscopic point of view, corresponds to the shear stiffness of the second gradient continuum? ? ? ?);

ii. damage corresponds to strain accumulation with a small increasing stress, hence the sharp decrease in Fig. ??.

The next section introduces the damage model adopted in this study.

\section{Numerical analysis and implementation of damage}

In this section, numerical examples are presented to show the applicability of the proposed approach by comparison with experimental evidence. To this end, shear tests of two different pantographic structures were numerically studied by modeling the energy 
terms in the commercial FE software COMSOL Multiphysics ${ }^{\circledR}$ utilizing its Weak Form PDE interface to implement the variational statement.

A pantographic layer consisting of standard pivots is first studied. The structure has beams of width $a=0.8 \mathrm{~mm}$ and depth $b=0.8 \mathrm{~mm}$, as well as standard pivots of radius $r=0.125 \mathrm{~mm}$ and height $h=0.6 \mathrm{~mm}$. The number of fibers along its width $\left(L_{b}\right)$ is $N_{f}=15$. The layer has a length of $L_{a}=90 \mathrm{~mm}$ and width of $L_{b}=30 \mathrm{~mm}$. The tested specimen was fabricated with Selective Laser Melting (SLM) technology. The stiffness parameters used in the numerical simulations are listed in Table. ??.

Table 1. Stiffness parameters used in the numerical simulations

\begin{tabular}{cc}
\hline Stiffness parameter & Numerical value \\
\hline$K_{e}$ & $5.79 \times 10^{5} \mathrm{~N} / \mathrm{m}$ \\
$K_{b g}$ & $0.77 \mathrm{Nm}$ \\
$K_{b n}$ & $0.77 \mathrm{Nm}$ \\
$K_{t}$ & $1.22 \mathrm{Nm}$ \\
$K_{s h}$ & $64000 \mathrm{~N} / \mathrm{m}$ \\
\hline
\end{tabular}

A numerical shear test was carried out by applying a displacement boundary condition on one of its shorter sides of the layer. In Fig. ??, the computed deformed shapes are presented for selected prescribed displacements, namely, $u_{0}=10,20,30,40$ and 50 $\mathrm{mm}$, and the results are compared with the experimentally observed deformed shapes. Each deformed shape has a contour plot representing out-of-plane displacements. The predicted deformed shapes compare very well with those obtained in the experimental tests. As expected, in the beginning of the shear test (especially, when prescribed displacement is equal to $10 \mathrm{~mm}$ ), the out-of-plane displacement is very low. However, for increasing values of the prescribed displacement, out-of-plane motions are observed and reach a maximum value of $5 \mathrm{~mm}$ in the vicinity of longer sides of the layer. 


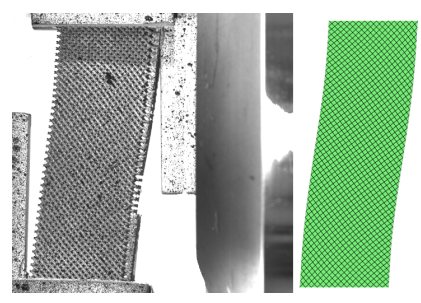

(a) $u=10 \mathrm{~mm}$

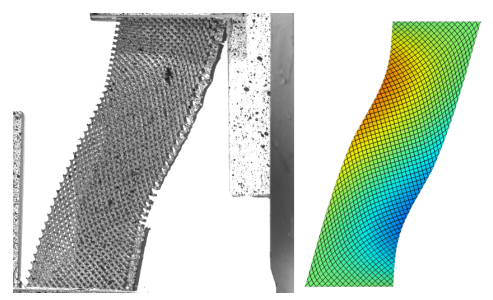

(c) $u=30 \mathrm{~mm}$

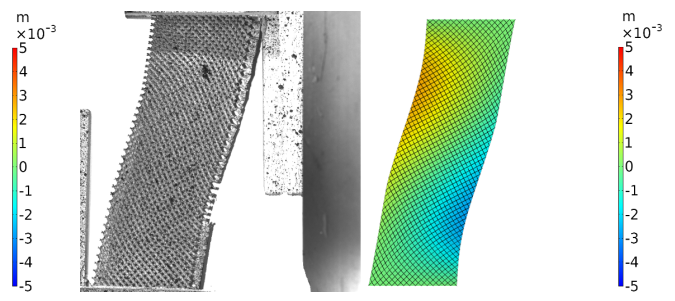

(b) $u=20 \mathrm{~mm}$
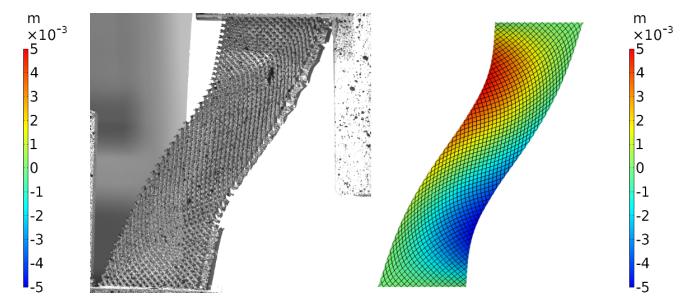

(d) $u=40 \mathrm{~mm}$
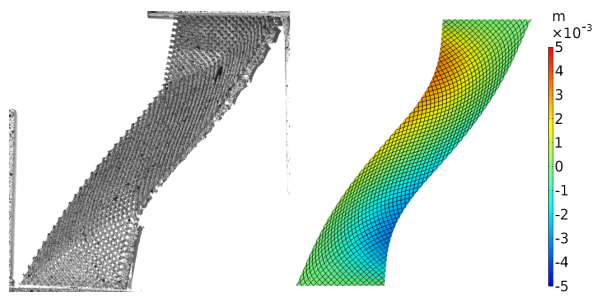

(e) $u=50 \mathrm{~mm}$

Figure 7. Out-of-plane displacements obtained by numerical simulations of a pantographic layer with standard pivots; pictures of experimental tests are also provided for comparison purposes.

In the second case, a pantographic layer with quasi-perfect pivots is considered. The structure has beams of width $a=1 \mathrm{~mm}$ and depth $b=1 \mathrm{~mm}$, as well as standard pivots of radius $r=0.4 \mathrm{~mm}$ and height $h=1.5 \mathrm{~mm}$. The number of fibers along its width $\left(L_{b}\right)$ is $N_{f}=7$. As quasi-perfect pivots were utilized to reduce out-of-plane deformations, the stiffness of the pivot defined in the numerical model was reduced (4 times) while other stiffnesses are kept identical.

In Fig. ??, the predicted deformed shapes are shown for prescribed displacements $u_{0}=$ 10, 20,30, 40 and $50 \mathrm{~mm}$, respectively. The effect of quasi-perfect pivots is significant and out-of-plane motions are considerably reduced compared to the previous case (Fig. ??). This investigation shows that the proposed numerical model predicts quite well the deformed shapes including the effect of out-of-plane deformations. 


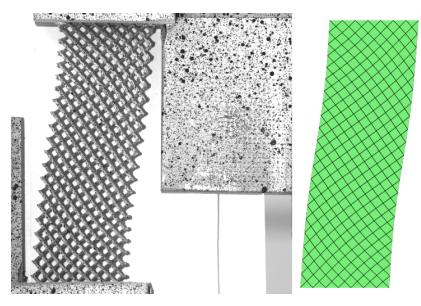

(a) $u=10 \mathrm{~mm}$

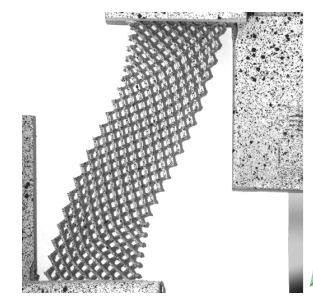

(c) $u=30 \mathrm{~mm}$

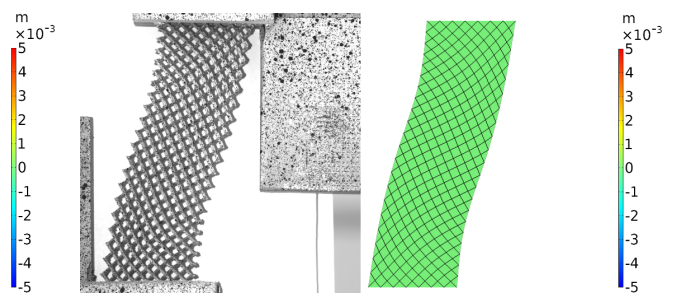

(b) $u=20 \mathrm{~mm}$

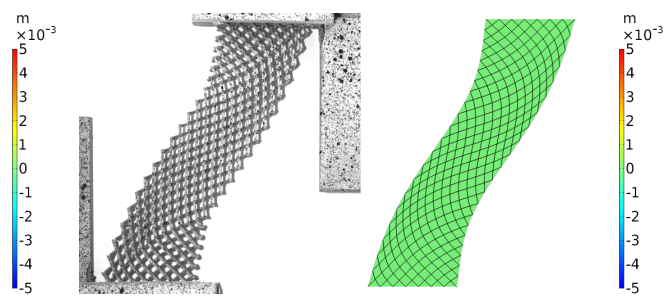

(d) $u=40 \mathrm{~mm}$
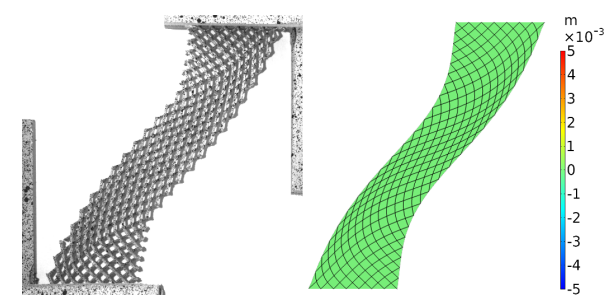

(e) $u=50 \mathrm{~mm}$

Figure 8. Out-of-plane displacements obtained by numerical simulations of a pantographic layer with quasi-perfect pivots; pictures of experimental tests are also provided for comparison purposes.

\section{Introduction of damage}

The crucial point concerns the fact that, at least in the first part of loading, the global deformation of the structure is dominated by the shear term, i.e. that related to torsion of the pivots. For this reason, in order to obtain simulations consistent with the measurements shown in Fig. ??, which show the occurrence of a softening mechanism that decreases the slope of the reaction force curve, a hypothesis was formulated according to which damage is actually occurring. A degradation law is introduced for the shear stiffness beyond a certain threshold depending on the angle $\gamma$ between the fibers (see Fig. ??). 

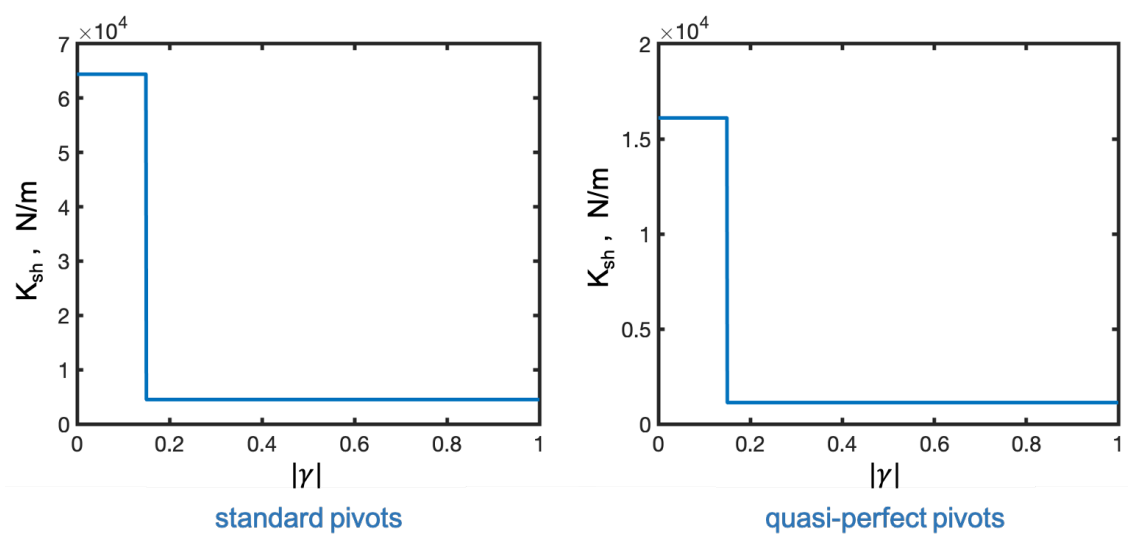

Figure 9. Threshold for damage growth. Standard pivots (left); quasi-perfect pivots (right).

Specifically, the displacement was selected at the yield point $u^{*}$ (i.e. when the slope of the curve changes due to inelasticity) and was obtained from the maximum value of the relative angle between the fibers of the two families. This angle is associated with the torsional deformation of the pivots. In correspondence with such angle, damage is activated (Fig. ??). When the angle in a point of the structure reaches this threshold, the shear stiffness $K_{s h}$ is reduced to a lower value shown in Fig. ??. This reduced value was obtained by comparing numerical simulations with the experimental data (see Fig. ??).

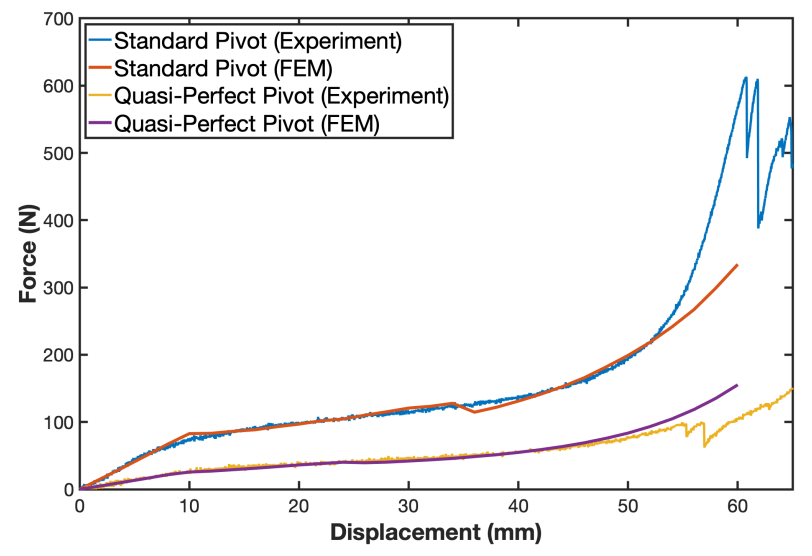

Figure 10. Force-displacement response in shear. Comparison between numerical and experimental measurements for the two specimens.

In Fig. ??, the experimentally measured force-displacement plots of both pantographic layers are compared to numerical simulations. The comparisons show a good match with the calibrated stiffnesses and the simplified damage-like approach. The experimental data 
show that damage occurring during the experiments becomes dominant after $u_{0}=60$ $\mathrm{mm}$, where the force-displacement curves have some jumps. Due to the simplified approach, it is also observed that the numerical force-displacement curves have jumps indicating that the damage occurred to specimen during simulations. Damaged portions of the specimens predicted by the simulations are shown in Fig. ??. When the prescribed displacement increases the total damaged area increases as well.
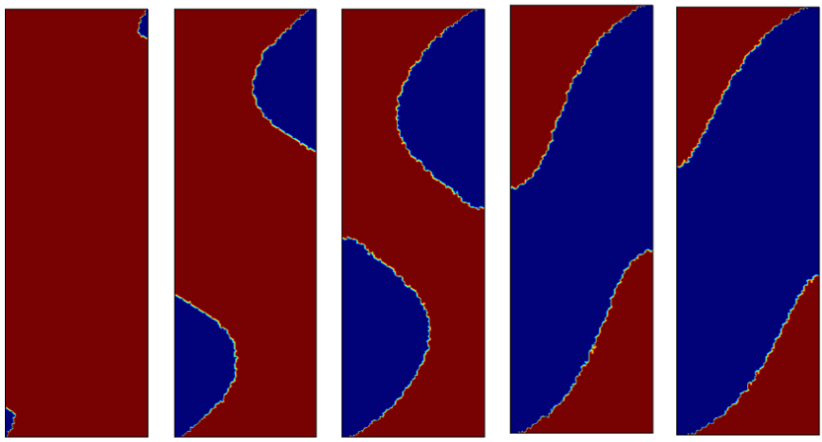

(a) standard pivots
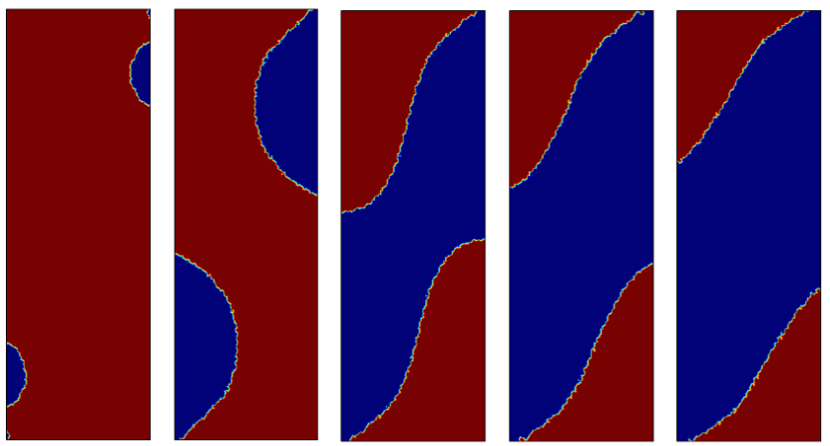

(b) quasi-perfect pivots

Figure 11. Extension of damaged portions of the specimen due to the simplified approach for both standard and quasi-perfect pivots for selected values of prescribed displacement $(10 \mathrm{~mm} ; 20 \mathrm{~mm} ; 30 \mathrm{~mm} ; 40 \mathrm{~mm} ; 50 \mathrm{~mm})$. Blue regions show reduced value of the shear stiffness, corresponding to damaged portions of the specimens.

In Figs. ??-??, the numerically computed angle change between fibers of the two families is plotted. These images show the regions were, in the real specimens, the hinges will be subjected to maximum values of torsion. After specific values of these relative angles, i.e. of torsion of the hinges, corresponding to the threshold values shown 
in Fig. ??, the shear stiffnesses degraded and the damage fields represented in Fig. ?? are obtained.
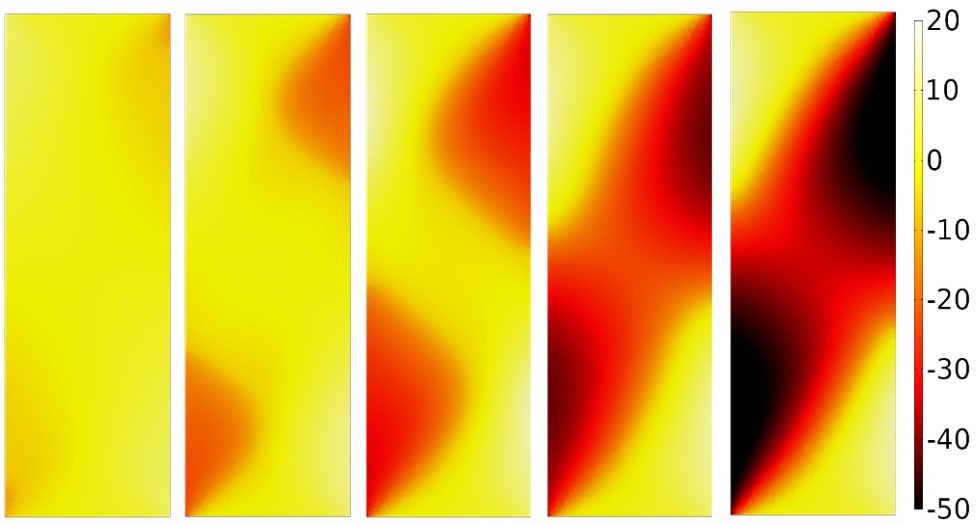

Figure 12. Numerically computed angle (in degrees) change between fibers of the two families for selected values of prescribed displacement $(10 \mathrm{~mm} ; 20 \mathrm{~mm} ; 30 \mathrm{~mm} ; 40 \mathrm{~mm}$; $50 \mathrm{~mm}$ ). Standard pivots case.
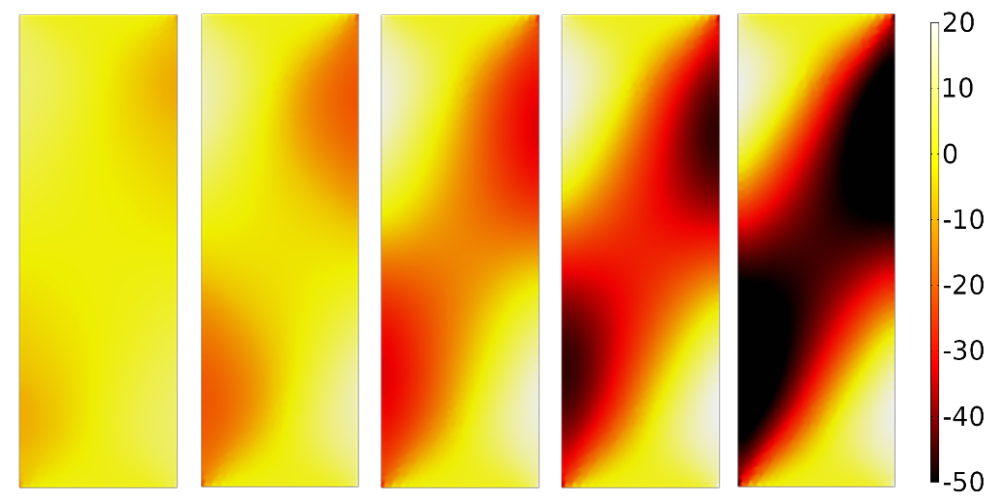

Figure 13. Numerically computed angle (in degrees) change between fibers of the two families for selected values of prescribed displacement $(10 \mathrm{~mm} ; 20 \mathrm{~mm} ; 30 \mathrm{~mm} ; 40 \mathrm{~mm}$; $50 \mathrm{~mm}$ ). Quasi-perfect pivots case.

\section{Digital Image Correlation analyses}

Digital Image Correlation (DIC ? ) can be used to analyze experimental images. For example, it can be employed to measure displacement and strain fields at prescribed resolution of a deformed specimen. This technique has been successfully applied several times to extract the displacement and strain fields in experiments involving

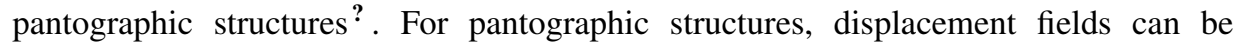


measured at macroscopic, mesoscopic and discrete scales. These scales are typical in any metamaterial model: in fact, when dealing with homogenization procedures one need to consider a microscopic or discrete scale (the architecture or microstructure of the metamaterial) and, at least, a macroscopic scale for the continuum homogenized model. Moreover, for pantographic structures, it is possible to introduce also a mesoscale in which each structural element can be described in terms of a continuum model, namely the beam model for the fibers (see e.g. ${ }^{\text {? }}$ for an efficient formulation). These displacement fields can then be compared with those predicted by numerical simulations. By this comparison, it is possible to validate the considered constitutive model.

The full validation of the models derived herein is beyond the scope of the present work. The kinematic fields provided by DIC analyses will be qualitatively compared with their numerical counterparts. Similarly, the calibration of the model parameters was performed by using the macroscopic load vs. displacement curves (Fig. ??). Since two models were derived at different scales, DIC analyses were also performed at two different scales by tailoring the kinematic bases and hypotheses. First, in macroscale analyses, continuous displacements were sought with exactly the same FE discretization as that used in numerical simulations. This is made possible thanks to the FE formulation available in the Correli 3.0 framework ${ }^{\text {? }}$. Second, mesoscale analyses were conducted in which the two families of beams were explicitly meshed, and each hinge was accounted for by assuming no displacement jump but allowing relative rotations to occur. Thus, it was possible to evaluate these rotations by following the angular changes at each hinge. This type of formulation was also implemented within the Correli 3.0 framework ${ }^{\text {? }}$. As proposed in previous studies? , a backtracking procedure was adopted to reposition the nominal mesoscale mesh onto the experimental reference configuration.

\section{Comparison between computed and DIC measured strain fields}

In the previous section, deformed shapes, experimental and numerical forcedisplacement curves were compared, and it was showed that findings were in a good agreement. As a further study, DIC provided displacement and strain fields, and these fields are compared to those obtained numerically.

In Figs. ??-??, DIC results (2-D displacement and strain fields) of the pantographic layer with standard pivots are presented for prescribed displacement values, namely $u=10,20,30,40$ and $50 \mathrm{~mm}$, respectively. The comparisons show quite good agreement and the discrepancies are in an acceptable range. In particular, displacements fields $\left(u_{x}\right.$ and $\left.u_{y}\right)$ are very close. Similarly, normal strains $\left(\varepsilon_{x x}, \varepsilon_{y y}\right)$ obtained by DIC are consistent with those obtained by numerical simulations, showing that the second gradient model along with the adopted damage model works quite well to investigate the mechanical behavior of metallic pantographic structures. It must be noticed that out-of-plane deformations that may occur in the specimen are not accounted for in DIC analyses, and that is why the differences between DIC and numerical analyses may become larger with increasing prescribed displacements. This can be seen in the comparisons provided in Figs. ?? and ?? (when the prescribed displacement is 40 and 50 
$\mathrm{mm}$, respectively). Although the deformed shapes compared each other very well, strains are slightly different.

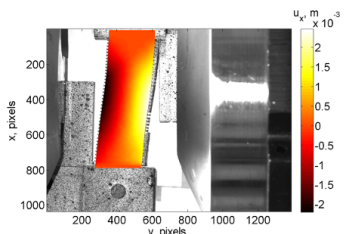

(a) $u_{x}$

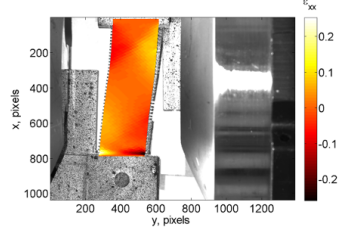

(c) $\varepsilon_{x x}$

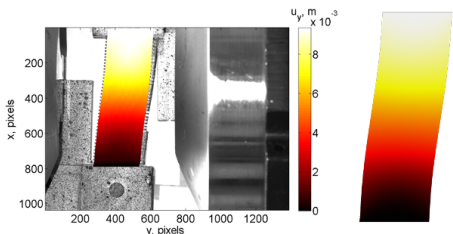

(b) $u_{y}$

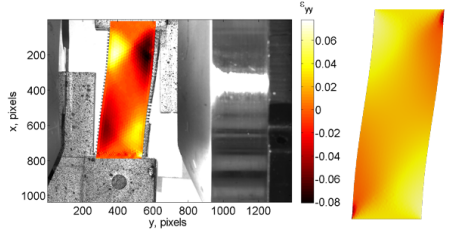

(d) $\varepsilon_{y y}$

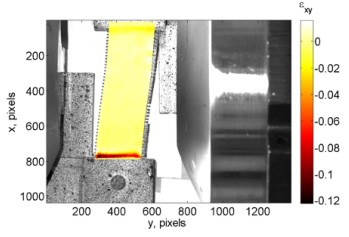

(e) $\varepsilon_{x y}$

Figure 14. Displacement $\left(u_{x}\right.$ and $\left.u_{y}\right)$ and strain $\left(\varepsilon_{x x}, \varepsilon_{y y}, \varepsilon_{x y}\right)$ fields obtained by DIC (left) and numerical (right) analyses for a layer with standard pivots $\left(u_{0}=10 \mathrm{~mm}\right)$. 


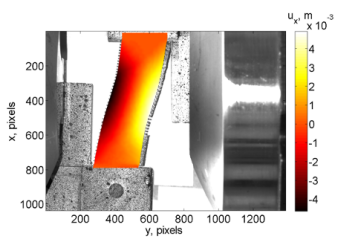

(a) $u_{x}$

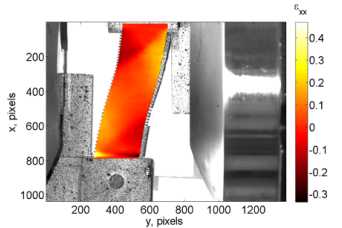

(c) $\varepsilon_{x x}$
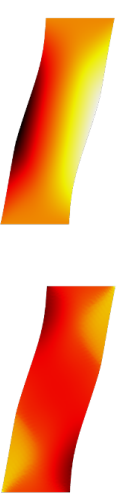$$
\text { . }
$$

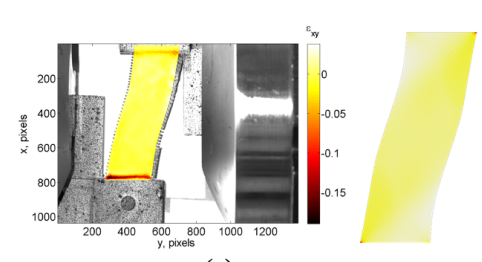

(e) $\varepsilon_{x y}$

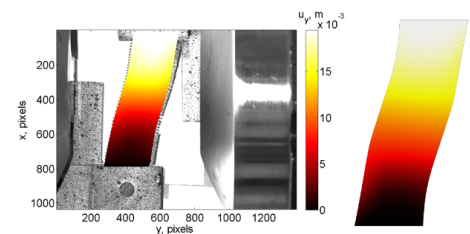

(b) $u_{y}$

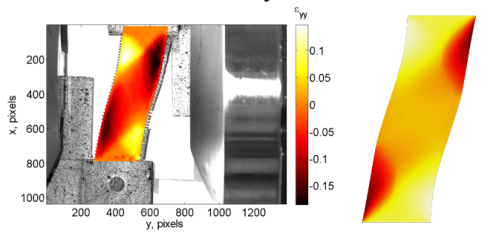

(d) $\varepsilon_{y y}$

Figure 15. Displacement $\left(u_{x}\right.$ and $\left.u_{y}\right)$ and strain $\left(\varepsilon_{x x}, \varepsilon_{y y}, \varepsilon_{x y}\right)$ fields obtained by DIC (left) and numerical (right) analyses for a layer with standard pivots $\left(u_{0}=20 \mathrm{~mm}\right)$. 


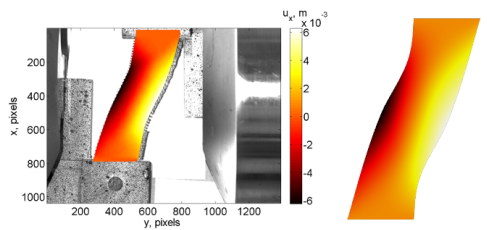

(a) $u_{x}$

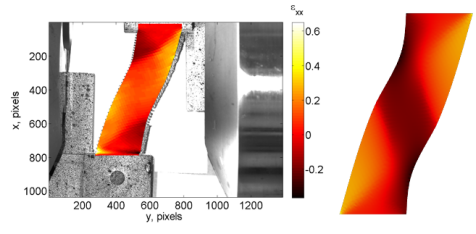

(c) $\varepsilon_{x x}$

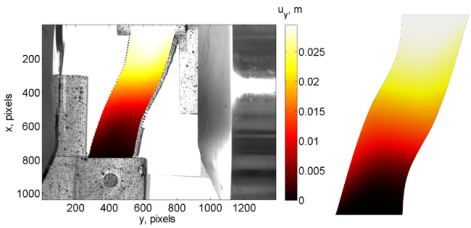

(b) $u_{y}$

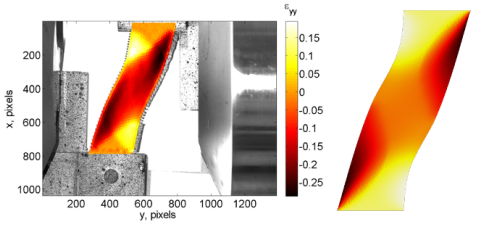

(d) $\varepsilon_{y y}$

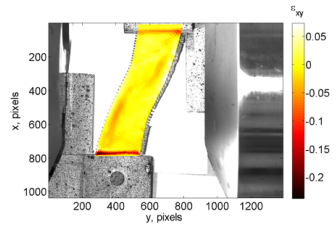

(e) $\varepsilon_{x y}$

Figure 16. Displacement $\left(u_{x}\right.$ and $\left.u_{y}\right)$ and strain $\left(\varepsilon_{x x}, \varepsilon_{y y}, \varepsilon_{x y}\right)$ fields obtained by DIC (left) and numerical (right) analyses for a layer with standard pivots $\left(u_{0}=30 \mathrm{~mm}\right)$. 


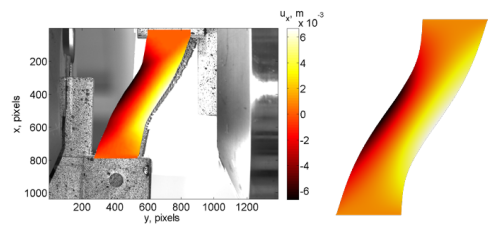

(a) $u_{x}$

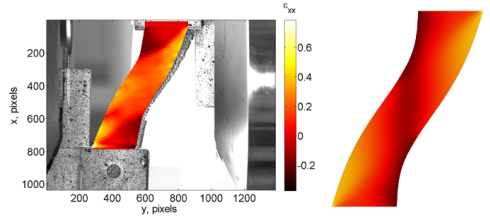

(c) $\varepsilon_{x x}$

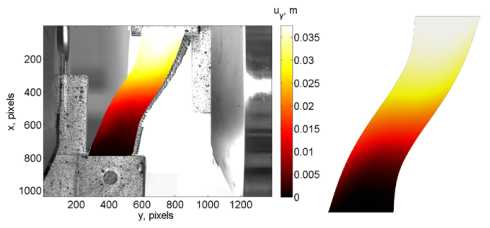

(b) $u_{y}$

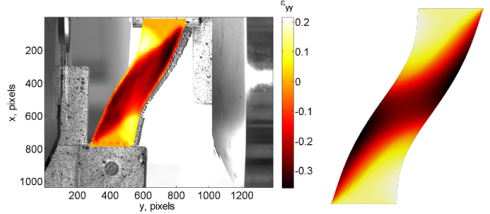

(d) $\varepsilon_{y y}$
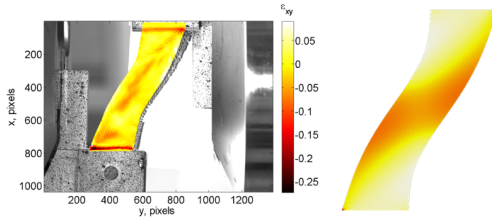

(e) $\varepsilon_{x y}$

Figure 17. Displacement $\left(u_{x}\right.$ and $\left.u_{y}\right)$ and strain $\left(\varepsilon_{x x}, \varepsilon_{y y}, \varepsilon_{x y}\right)$ fields obtained by DIC (left) and numerical (right) analyses for a layer with standard pivots $\left(u_{0}=40 \mathrm{~mm}\right)$. 


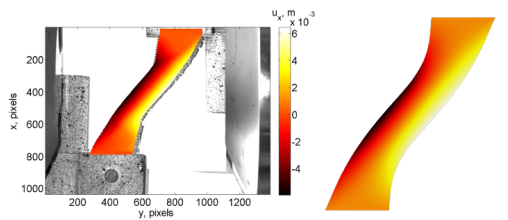

(a) $u_{x}$

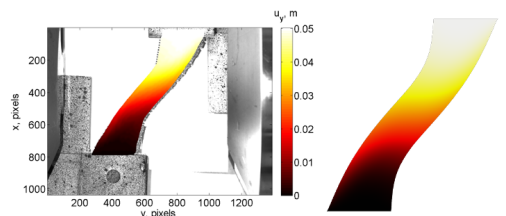

(b) $u_{y}$

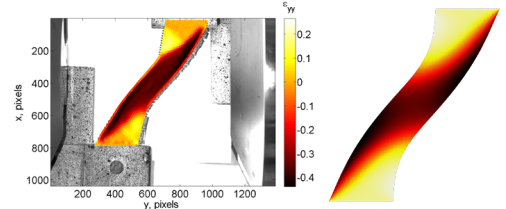

(d) $\varepsilon_{y y}$

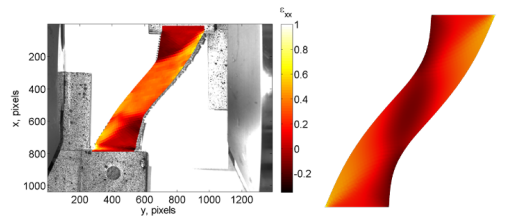

(c) $\varepsilon_{x x}$

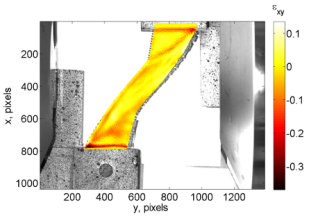

(e) $\varepsilon_{x y}$

Figure 18. Displacement $\left(u_{x}\right.$ and $\left.u_{y}\right)$ and strain $\left(\varepsilon_{x x}, \varepsilon_{y y}, \varepsilon_{x y}\right)$ fields obtained by DIC (left) and numerical (right) analyses for a layer with standard pivots $\left(u_{0}=50 \mathrm{~mm}\right)$.

Next, the computed results for the pantographic layer with quasi-perfect pivots are compared in Figs. ??-?? for prescribed displacements $u=10,20,30,40$ and $50 \mathrm{~mm}$, respectively. Similar to the findings obtained for the pantographic layer with standard pivots, the displacement and strains fields obtained by the numerical simulations compare well with those computed via DIC. As the out-of-plane deformations are alleviated via quasi-perfect pivots, better agreement is obtained between numerical and DIC results. 


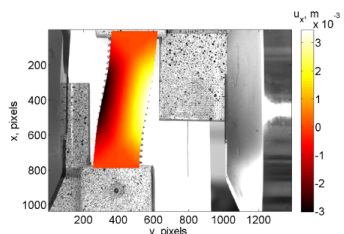

(a) $u_{x}$

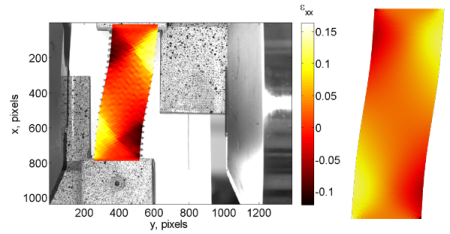

(c) $\varepsilon_{x x}$

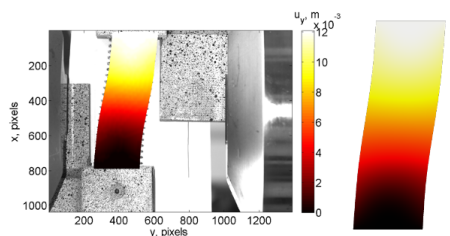

(b) $u_{y}$

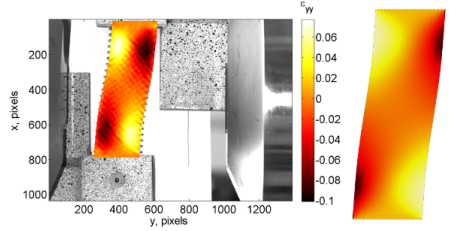

(d) $\varepsilon_{y y}$

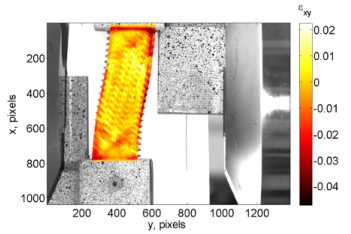

(e) $\varepsilon_{x y}$

Figure 19. Displacement $\left(u_{x}\right.$ and $\left.u_{y}\right)$ and strain $\left(\varepsilon_{x x}, \varepsilon_{y y}, \varepsilon_{x y}\right)$ fields obtained by both DIC (left) and numerical (right) analyses for layer with quasi-perfect pivots $\left(u_{0}=10 \mathrm{~mm}\right)$. 


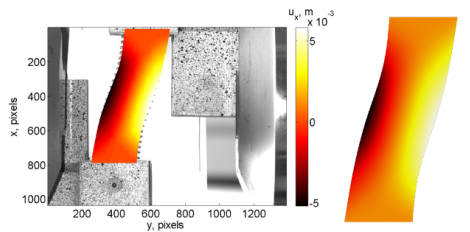

(a) $u_{x}$

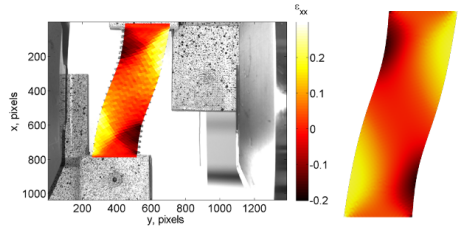

(c) $\varepsilon_{x x}$

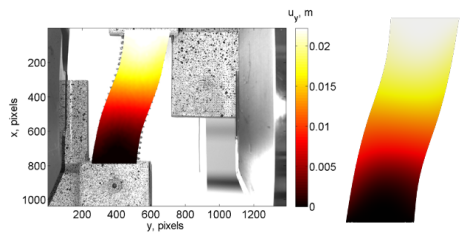

(b) $u_{y}$

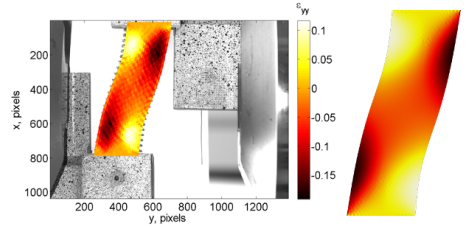

(d) $\varepsilon_{y y}$

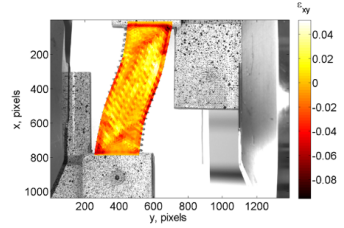

(e) $\varepsilon_{x y}$

Figure 20. Displacement $\left(u_{x}\right.$ and $\left.u_{y}\right)$ and strain $\left(\varepsilon_{x x}, \varepsilon_{y y}, \varepsilon_{x y}\right)$ fields obtained by both DIC (left) and numerical (right) analyses for layer with quasi-perfect pivots $\left(u_{0}=20 \mathrm{~mm}\right)$. 


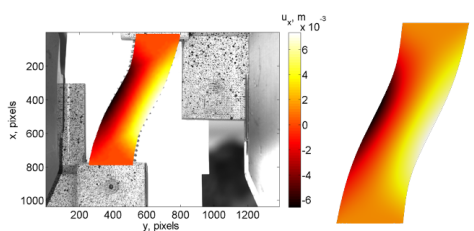

(a) $u_{x}$

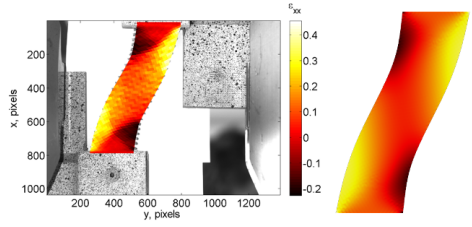

(c) $\varepsilon_{x x}$

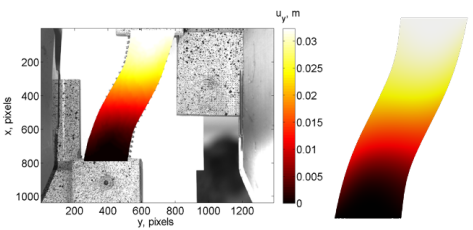

(b) $u_{y}$

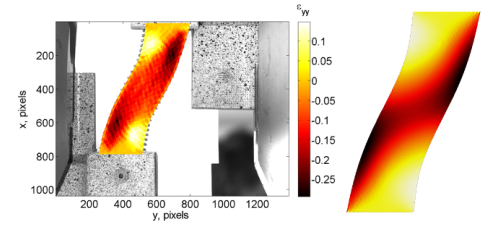

(d) $\varepsilon_{y y}$
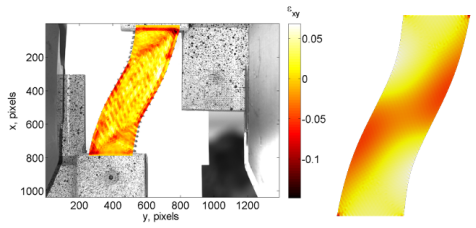

(e) $\varepsilon_{x y}$

Figure 21. Displacement $\left(u_{x}\right.$ and $\left.u_{y}\right)$ and strain $\left(\varepsilon_{x x}, \varepsilon_{y y}, \varepsilon_{x y}\right)$ fields obtained by both DIC (left) and numerical (right) analyses for layer with quasi-perfect pivots $\left(u_{0}=30 \mathrm{~mm}\right)$. 


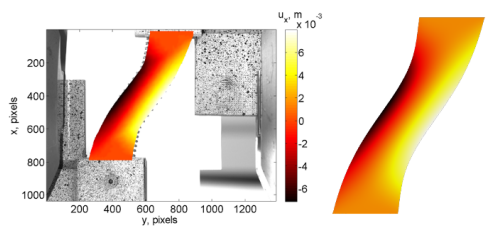

(a) $u_{x}$

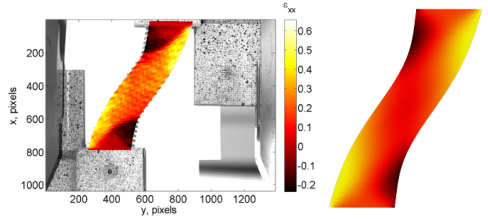

(c) $\varepsilon_{x x}$

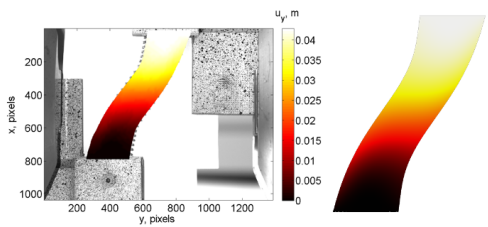

(b) $u_{y}$

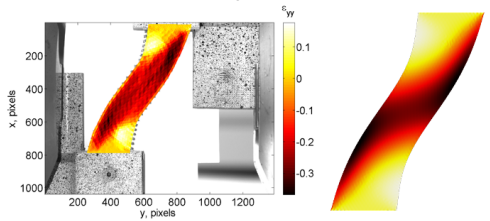

(d) $\varepsilon_{y y}$
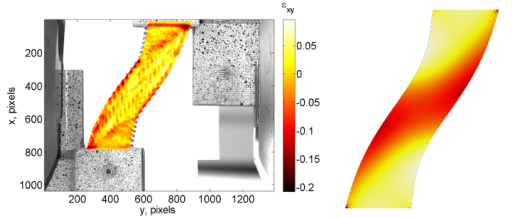

(e) $\varepsilon_{x y}$

Figure 22. Displacement $\left(u_{x}\right.$ and $\left.u_{y}\right)$ and strain $\left(\varepsilon_{x x}, \varepsilon_{y y}, \varepsilon_{x y}\right)$ fields obtained by both DIC (left) and numerical (right) analyses for layer with quasi-perfect pivots ( $u_{0}=40 \mathrm{~mm}$ ). 


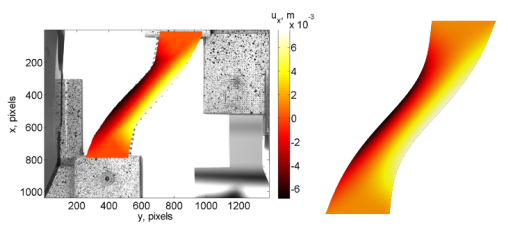

(a) $u_{x}$

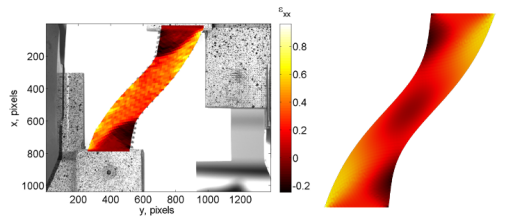

(c) $\varepsilon_{x x}$

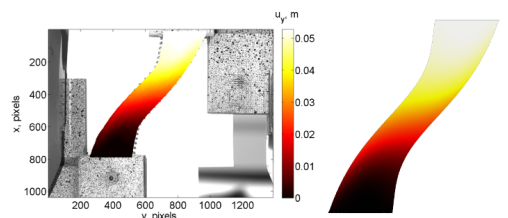

(b) $u_{y}$

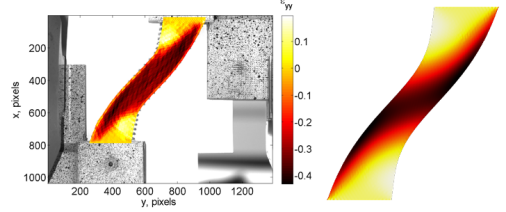

(d) $\varepsilon_{y y}$
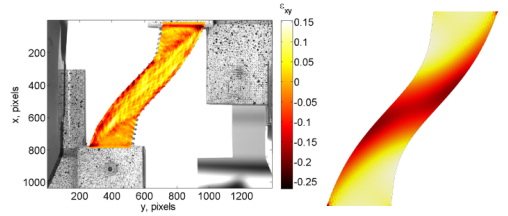

(e) $\varepsilon_{x y}$

Figure 23. Displacement $\left(u_{x}\right.$ and $\left.u_{y}\right)$ and strain $\left(\varepsilon_{x x}, \varepsilon_{y y}, \varepsilon_{x y}\right)$ fields obtained by both DIC (left) and numerical (right) analyses for layer with quasi-perfect pivots $\left(u_{0}=50 \mathrm{~mm}\right)$.

\section{Qualitative assessment of damage hypothesis}

The angle changes in correspondence of the hinges (see Figs. ??-??) were determined by microscale DIC. This type of information is crucial if the damage hypothesis has to be validated. If one compares the damage growth (Fig. ??) and the computed (Figs. ??-??) and measured (Figs. ??-??) rotations, it can be observed that the rotation field, measured for each hinge, behaves similarly to the computed one. One may argue that the measured rotations in the case of standard pivots specimen are large enough to produce damage also in the top-left and bottom-right areas, which is not predicted numerically. A first reason for this discrepancy is due to the fact that the simulations are run at the macroscale while the DIC measurements were performed at the microscale. Moreover, out-of-plane motions occurred in that experiment and precisely in those areas, which may bias the DIC results. The damage growth law is related to the angle change. As it is shown in Fig. ??, damage growth consists in a threshold law triggered on a specific value of the angle change. Consequently, the aforementioned portions remained undamaged according to the present model.

In Fig. ??, comparisons between DIC measured and computed values of angle changes for three specific points in the pantographic structures are shown. 


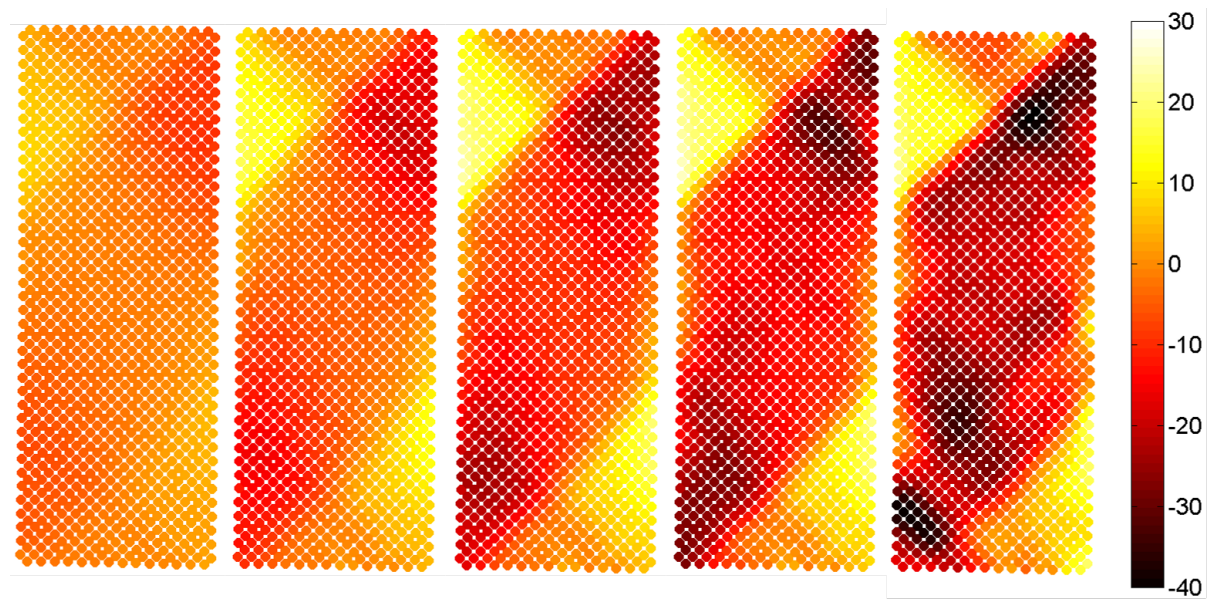

Figure 24. Discrete computation of rotations of connecting hinges for selected values of the prescribed displacement $(10 \mathrm{~mm} ; 20 \mathrm{~mm} ; 30 \mathrm{~mm} ; 40 \mathrm{~mm} ; 50 \mathrm{~mm})$. Standard pivots case.

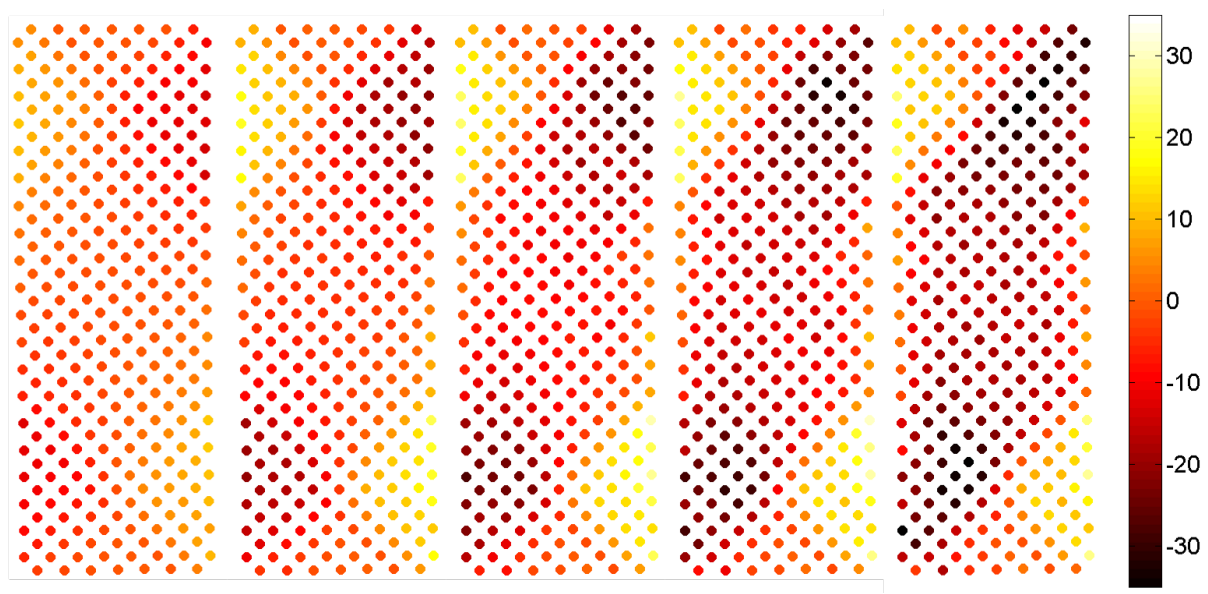

Figure 25. Discrete computation of rotations of connecting hinges for selected values of the prescribed displacement $(10 \mathrm{~mm} ; 20 \mathrm{~mm} ; 30 \mathrm{~mm} ; 40 \mathrm{~mm} ; 50 \mathrm{~mm})$. Quasi-perfect pivots case.

In Fig. ??, the angle changes for selected points are shown and compared when measured via DIC and computed with the model. The responses are qualitatively similar, but the most important information that can be obtained from these plots, both in case of DIC and of FE simulations, is that all angles (but particularly the central one) change considerably for values of the prescribed displacement corresponding to configurations where damage increases significantly (see Fig. ??). This observation also proves that the 
damage hypothesis chosen for describing the experimental observations is sufficient. In case of quasi-perfect pivots, this effect is lower than in case with standard pivots.
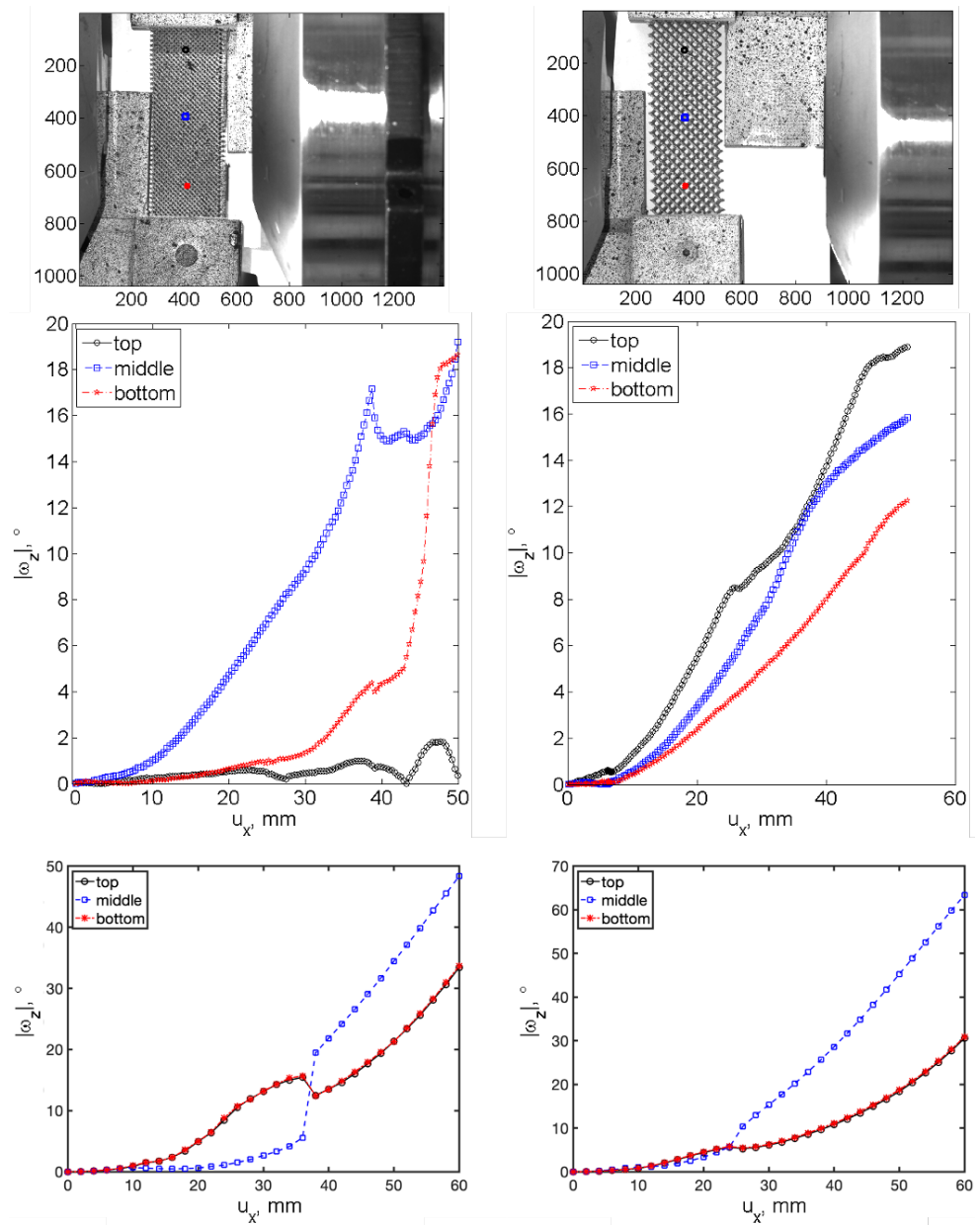

Figure 26. Angle changes for selected points (top) measured via DIC (middle) and computed in numerical simulations (bottom) for standard pivots case (left) and quasi-perfect pivots case (right). 


\section{Conclusive remarks}

In this work, the mechanical behaviour of a particular fibrous metamaterial, called pantographic metamaterial, was analyzed. The two shear tests show that in some cases out-of-plane deformations may be present. The main objective of this work was to determine the fundamental mechanisms related to out-of-plane deformations. It was discovered that the problem was non-trivial and involved instability phenomena. In order to deal with such phenomena, which in the present study were reported only in a qualitative way and checked by means of numerical analyses, it was necessary to introduce advanced mathematical tools such as those used in Refs. ????? . A further study will cover this type of analysis. Furthermore, the sensitivity to variation of the constitutive parameters of such out-of-plane instabilities needs to be rigorously investigated.

It is worth stressing that the present study has verified and confirmed how the mechanics of pantographic structures was strongly influenced by the micromechanics of its smallest structural elements, namely, the hinges, also known as pivots. These elements store most of the structure total strain energy. This conclusion is supported by the fact that, in order to model the change in slope of the experimental force-displacement curve (which is classically interpreted as being due to plasticity or damage phenomena, or to a combination of these) it was sufficient to introduce a damage criterion for pivots only.

A more detailed investigation needs to be carried out to correctly address thereof plasticity. For this purpose, useful tools have been developed?? . Furthermore, to introduce damage phenomena in a more rigorous way it may be useful to use the results obtained in Refs.??????

An important role in the deformation mechanisms of pantographic structures was played by hinges. So-called quasi-perfect pivots had the remarkable advantage of significantly decreasing the measured reaction force and, moreover, having a lower torsional stiffness than that associated with standard pivots. This property allowed out-ofplane deformations to be reduced. It is therefore very important to produce pantographic structures whose pivots behave as perfect hinges. This has successfully been achieved in the case of polyamide printed specimens? .

\section{Acknowledgments}

MS was supported by P.O.R. SARDEGNA F.S.E. 2014-2020 - Asse III "Istruzione e Formazione, Obiettivo Tematico: 10, Obiettivo Specifico: 10.5, Azione dell'accordo di Partenariato:10.5.12" Avviso di chiamata per il finanziamento di Progetti di ricerca Anno 2017.

\section{References}

1. D. George, C. Spingarn, C. Dissaux, M. Nierenberger, R. A. Rahman, and Y. Rémond, "Examples of multiscale and multiphysics numerical modeling of biological tissues", BioMedical Materials and Engineering, vol. 28, no. s1, pp. S15-S27, 2017. 
2. I. Giorgio, U. Andreaus, and A. Madeo, "The influence of different loads on the remodeling process of a bone and bioresorbable material mixture with voids", Continuum Mechanics and Thermodynamics, vol. 28, no. 1-2, pp. 21-40, 2016.

3. I. Giorgio, F. dell'Isola, U. Andreaus, F. Alzahrani, T. Hayat, and T. Lekszycki, "On mechanically driven biological stimulus for bone remodeling as a diffusive phenomenon", Biomechanics and Modeling in Mechanobiology, vol. 18, no. 6, pp. 1639-1663, 2019.

4. I. Giorgio, U. Andreaus, D. Scerrato, and P. Braidotti, "Modeling of a non-local stimulus for bone remodeling process under cyclic load: Application to a dental implant using a bioresorbable porous material", Mathematics and Mechanics of Solids, vol. 22, no. 9, pp. 1790-1805, 2017.

5. T. Lekszycki and N. Olhoff, "Optimal design of viscoelastic structures under forced steadystate vibration”, ASCE Journal of Structural Mechanics, vol. 9, no. 4, pp. 363-387, 1981.

6. L. Dietrich, T. Lekszycki, and K. Turski, "Problems of identification of mechanical characteristics of viscoelastic composites", Acta Mechanica, vol. 126, no. 1, pp. 153-167, 1998.

7. Z. Mróz and T. Lekszycki, "Optimal support reaction in elastic frame structures”, Computers \& Structures, vol. 14, no. 3-4, pp. 179-185, 1981.

8. Y. Lu and T. Lekszycki, "A novel coupled system of non-local integro-differential equations modelling Young's modulus evolution, nutrients' supply and consumption during bone fracture healing", Zeitschrift für Angewandte Mathematik und Physik, vol. 67, no. 5, pp. 112, 2016.

9. Y. Lu and T. Lekszycki, "New description of gradual substitution of graft by bone tissue including biomechanical and structural effects, nutrients supply and consumption", Continuum Mechanics and Thermodynamics, vol. 30, no. 5, pp. 995-1009, 2018.

10. I. Giorgio, U. Andreaus, F. dell'Isola, and T. Lekszycki, "Viscous second gradient porous materials for bones reconstructed with bio-resorbable grafts", Extreme Mechanics Letters, vol. 13, pp. 141-147, 2017.

11. D. Scerrato, A. M. Bersani, and I. Giorgio, "Bio-inspired design of a porous resorbable scaffold for bone reconstruction: A preliminary study", Biomimetics, vol. 6(1), no. 18, 2021.

12. F. dell'Isola, P. Seppecher, M. Spagnuolo, E. Barchiesi, F. Hild, T. Lekszycki, I. Giorgio, L. Placidi, U. Andreaus, M. Cuomo, et al., "Advances in pantographic structures: design, manufacturing, models, experiments and image analyses", Continuum Mechanics and Thermodynamics, vol. 31, no. 4, pp. 1231-1282, 2019.

13. E. Turco, F. dell'Isola, N. L. Rizzi, R. Grygoruk, W. H. Müller, and C. Liebold, "Fiber rupture in sheared planar pantographic sheets: Numerical and experimental evidence", Mechanics Research Communications, vol. 76, pp. 86-90, 2016.

14. M. E. Yildizdag, E. Barchiesi, and F. dell'Isola, "Three-point bending test of pantographic blocks: numerical and experimental investigation", Mathematics and Mechanics of Solids, vol. 25, no. 10, pp. 1965-1978, 2020.

15. K. Bertoldi, P. M. Reis, S. Willshaw, and T. Mullin, "Negative Poisson's ratio behavior induced by an elastic instability", Advanced Materials, vol. 22, no. 3, pp. 361-366, 2010.

16. C. Pideri and P. Seppecher, "A second gradient material resulting from the homogenization of an heterogeneous linear elastic medium", Continuum Mechanics and Thermodynamics, vol. 9, no. 5, pp. 241-257, 1997. 
17. L. Placidi, F. dell'Isola, and E. Barchiesi, "Heuristic homogenization of Euler and pantographic beams", in Mechanics of Fibrous Materials and Applications (J. F. G. C. Picu, ed.), pp. 123-155, Springer, Cham, Switzerland, 2020.

18. M. Pingaro, E. Reccia, and P. Trovalusci, "Homogenization of random porous materials with low-order virtual elements", ASCE-ASME Journal of Risk and Uncertainty in Engineering Systems, Part B: Mechanical Engineering, vol. 5, no. 3, pp. 030905-1-10, 2019.

19. E. Reccia, M. L. De Bellis, P. Trovalusci, and R. Masiani, "Sensitivity to material contrast in homogenization of random particle composites as micropolar continua", Composites Part B: Engineering, vol. 136, pp. 39-45, 2018.

20. J. Torabi and J. Niiranen, "Microarchitecture-dependent nonlinear bending analysis for cellular plates with prismatic corrugated cores via an anisotropic strain gradient plate theory of firstorder shear deformation”, Engineering Structures, vol. 236, no. 112117, 2021.

21. A. Madeo, A. Della Corte, I. Giorgio, and D. Scerrato, "Modeling and designing micro-and nano-structured metamaterials: towards the application of exotic behaviors", Mathematics and Mechanics of Solids, vol. 22, no. 4, pp. 873-884, 2017.

22. F. dell' Isola and D. J. Steigmann, Discrete and Continuum Models for Complex Metamaterials. Cambridge University Press, Cambridge, 2020.

23. E. Barchiesi, M. Spagnuolo, and L. Placidi, "Mechanical metamaterials: a state of the art", Mathematics and Mechanics of Solids, vol. 24, no. 1, pp. 212-234, 2019.

24. B. E. Abali and H. Yang, "Parameter determination of metamaterials in generalized mechanics as a result of computational homogenization", in Advanced Problems in Mechanics. APM 2019. Lecture Notes in Mechanical Engineering (A. K. A. D. Indeitsev, ed.), pp. 22-31, Springer, Cham, Switzerland, 2019.

25. A. Carcaterra, F. dell'Isola, R. Esposito, and M. Pulvirenti, "Macroscopic description of microscopically strongly inhomogenous systems: A mathematical basis for the synthesis of higher gradients metamaterials", Archive for Rational Mechanics and Analysis, vol. 218, no. 3, pp. 1239-1262, 2015.

26. F. dell'Isola, E. Barchiesi, and A. Misra, "Naive model theory: Its applications to the theory of metamaterials design", in Discrete and Continuum Models for Complex Metamaterials (F. dell'Isola and D. Steigmann, eds.), pp. 141-196, Cambridge University Press, Cambridge, 2020.

27. S. Eugster, F. dell'Isola, and D. Steigmann, "Continuum theory for mechanical metamaterials with a cubic lattice substructure", Mathematics and Mechanics of Complex Systems, vol. 7, no. 1, pp. 75-98, 2019.

28. I. Giorgio, A. Ciallella, and D. Scerrato, "A study about the impact of the topological arrangement of fibers on fiber-reinforced composites: Some guidelines aiming at the development of new ultra-stiff and ultra-soft metamaterials", International Journal of Solids and Structures, vol. 203, pp. 73-83, 2020.

29. S. Khakalo and J. Niiranen, "Lattice structures as thermoelastic strain gradient metamaterials: Evidence from full-field simulations and applications to functionally step-wise-graded beams", Composites Part B: Engineering, vol. 177, p. 107224, 2019.

30. D. Scerrato and I. Giorgio, "Equilibrium of two-dimensional cycloidal pantographic metamaterials in three-dimensional deformations", Symmetry, vol. 11(12), no. 1523, 2019. 
31. M. Spagnuolo and A. M. Cazzani, "Contact interactions in complex fibrous metamaterials", Continuum Mechanics and Thermodynamics, pp. 1-17, 2021, DOI:10.1007/s00161-02101018-y.

32. M. Spagnuolo, K. Barcz, A. Pfaff, F. dell'Isola, and P. Franciosi, "Qualitative pivot damage analysis in aluminum printed pantographic sheets: numerics and experiments", Mechanics Research Communications, vol. 83, pp. 47-52, 2017.

33. M. Spagnuolo, P. Peyre, and C. Dupuy, "Phenomenological aspects of quasi-perfect pivots in metallic pantographic structures", Mechanics Research Communications, vol. 101, no. $103415,2019$.

34. E. Turco, A. Misra, M. Pawlikowski, F. dell'Isola, and F. Hild, "Enhanced Piola-Hencky discrete models for pantographic sheets with pivots without deformation energy: numerics and experiments", International Journal of Solids and Structures, vol. 147, pp. 94-109, 2018.

35. A. Misra, T. Lekszycki, I. Giorgio, G. Ganzosch, W. H. Müller, and F. dell'Isola, "Pantographic metamaterials show atypical Poynting effect reversal", Mechanics Research Communications, vol. 89, pp. 6-10, 2018.

36. I. Giorgio, N. L. Rizzi, U. Andreaus, and D. J. Steigmann, "A two-dimensional continuum model of pantographic sheets moving in a 3D space and accounting for the offset and relative rotations of the fibers", Mathematics and Mechanics of Complex Systems, vol. 7, no. 4, pp. 311-325, 2019.

37. E. Turco, M. Golaszewski, A. Cazzani, and N. L. Rizzi, "Large deformations induced in planar pantographic sheets by loads applied on fibers: experimental validation of a discrete Lagrangian model", Mechanics Research Communications, vol. 76, pp. 51-56, 2016.

38. J.-J. Alibert, P. Seppecher, and F. dell'Isola, "Truss modular beams with deformation energy depending on higher displacement gradients", Mathematics and Mechanics of Solids, vol. 8, no. 1, pp. 51-73, 2003.

39. P. Seppecher, J.-J. Alibert, and F. dell'Isola, "Linear elastic trusses leading to continua with exotic mechanical interactions", Journal of Physics: Conference Series, vol. 319, no. 1, p. 012018, 2011.

40. F. dell'Isola, I. Giorgio, M. Pawlikowski, and N. L. Rizzi, "Large deformations of planar extensible beams and pantographic lattices: heuristic homogenization, experimental and numerical examples of equilibrium", Proceedings of the Royal Society A: Mathematical, Physical and Engineering Sciences, vol. 472(2185), no. 20150790, 2016.

41. E. Barchiesi, S. R. Eugster, F. dell'Isola, and F. Hild, "Large in-plane elastic deformations of bi-pantographic fabrics: asymptotic homogenization and experimental validation", Mathematics and Mechanics of Solids, vol. 25, no. 3, pp. 739-767, 2020.

42. E. Barchiesi, F. dell'Isola, F. Hild, and P. Seppecher, "Two-dimensional continua capable of large elastic extension in two independent directions: asymptotic homogenization, numerical simulations and experimental evidence", Mechanics Research Communications, vol. 103, no. $103466,2020$.

43. F. dell'Isola, M. Cuomo, L. Greco, and A. Della Corte, "Bias extension test for pantographic sheets: numerical simulations based on second gradient shear energies", Journal of Engineering Mathematics, vol. 103, no. 1, pp. 127-157, 2017. 
44. V. A. Eremeyev, F. dell'Isola, C. Boutin, and D. Steigmann, "Linear pantographic sheets: existence and uniqueness of weak solutions", Journal of Elasticity, vol. 132, no. 2, pp. 175196, 2018.

45. E. Turco, F. dell'Isola, A. Cazzani, and N. L. Rizzi, "Hencky-type discrete model for pantographic structures: numerical comparison with second gradient continuum models", Zeitschrift für Angewandte Mathematik und Physik, vol. 67, no. 85, 2016.

46. E. Turco and N. L. Rizzi, "Pantographic structures presenting statistically distributed defects: numerical investigations of the effects on deformation fields", Mechanics Research Communications, vol. 77, pp. 65-69, 2016.

47. M. Spagnuolo, M. E. Yildizdag, U. Andreaus, and A. M. Cazzani, "Are higher-gradient models also capable of predicting mechanical behavior in the case of wide-knit pantographic structures?", Mathematics and Mechanics of Solids, vol. 26, no. 1, pp. 18-29, 2021.

48. I. Giorgio, N. Rizzi, and E. Turco, "Continuum modelling of pantographic sheets for out-ofplane bifurcation and vibrational analysis", Proceedings of the Royal Society A: Mathematical, Physical and Engineering Sciences, vol. 473, no. 2207, p. 20170636, 2017.

49. P. Germain, "La méthode des puissances virtuelles en mécanique des milieux continus, premiere partie: théorie du second gradient", Journal de Mécanique, vol. 12, no. 2, pp. 235274, 1973.

50. P. Germain, "The method of virtual power in the mechanics of continuous media, I: Secondgradient theory", Mathematics and Mechanics of Complex Systems, vol. 8, no. 2, pp. 153-190, 2020.

51. P. Germain, "The method of virtual power in continuum mechanics. Part 2: Microstructure", SIAM Journal on Applied Mathematics, vol. 25, no. 3, pp. 556-575, 1973.

52. L. Lebedev, M. Cloud, and V. Eremeyev, Tensor Analysis with Applications in Continuum Mechanics. World Scientific, Singapore, 2018.

53. B. Desmorat, M. Spagnuolo, and E. Turco, "Stiffness optimization in nonlinear pantographic structures", Mathematics and Mechanics of Solids, vol. 25, no. 12, pp. 2252-2262, 2020.

54. F. Maurin, F. Greco, and W. Desmet, "Isogeometric analysis for nonlinear planar pantographic lattice: discrete and continuum models", Continuum Mechanics and Thermodynamics, vol. 31, no. 4, pp. 1051-1064, 2019.

55. U. Andreaus, M. Spagnuolo, T. Lekszycki, and S. R. Eugster, "A Ritz approach for the static analysis of planar pantographic structures modeled with nonlinear Euler-Bernoulli beams", Continuum Mechanics and Thermodynamics, vol. 30, no. 5, pp. 1103-1123, 2018.

56. M. A. Sutton, J. J. Orteu, and H. Schreier, Image correlation for shape, motion and deformation measurements: basic concepts, theory and applications. Springer, Berlin, 2009.

57. F. Hild and S. Roux, "Digital image correlation", in Optical methods for solid mechanics: a fullfield approach (E. H. P. Rastogi, ed.), Wiley, Weinheim, 2012.

58. H. Leclerc, J. Neggers, F. Mathieu, F. Hild, and S. Roux, Correli 3.0. IDDN.FR.001.520008.000.S.P.2015.000.31500, Agence pour la Protection des Programmes, Paris (France), 2015.

59. E. Barchiesi, F. dell'Isola, and F. Hild, "On the validation of homogenized modeling for bipantographic metamaterials via Digital Image Correlation", International Journal of Solids and Structures, vol. 208-209, pp. 49-62, 2021. 
60. M. Cuomo, F. dell'Isola, L. Greco, and N. Rizzi, "First versus second gradient energies for planar sheets with two families of inextensible fibres: investigation on deformation boundary layers, discontinuities and geometrical instabilities", Composites Part B: Engineering, vol. 115, pp. 423-448, 2017.

61. V. A. Eremeyev and H. Altenbach, "Equilibrium of a second-gradient fluid and an elastic solid with surface stresses", Meccanica, vol. 49, no. 11, pp. 2635-2643, 2014.

62. D. Scerrato, I. Giorgio, and N. L. Rizzi, "Three-dimensional instabilities of pantographic sheets with parabolic lattices: numerical investigations", Zeitschrift für Angewandte Mathematik und Physik, vol. 67(3), no. 53, 2016.

63. E. Barchiesi, G. Ganzosch, C. Liebold, L. Placidi, R. Grygoruk, and W. H. Müller, "Out-ofplane buckling of pantographic fabrics in displacement-controlled shear tests: Experimental results and model validation", Continuum Mechanics and Thermodynamics, vol. 31, no. 1, pp. 33-45, 2019.

64. S. Khakalo, V. Balobanov, and J. Niiranen, "Modelling size-dependent bending, buckling and vibrations of 2D triangular lattices by strain gradient elasticity models: applications to sandwich beams and auxetics", International Journal of Engineering Science, vol. 127, pp. 33-52, 2018.

65. H. Altenbach and V. Eremeyev, "Strain rate tensors and constitutive equations of inelastic micropolar materials", International Journal of Plasticity, vol. 63, pp. 3-17, 2014.

66. A. Bertram, "Finite gradient elasticity and plasticity: a constitutive mechanical framework", Continuum Mechanics and Thermodynamics, vol. 27, no. 6, pp. 1039-1058, 2015.

67. A. Misra and P. Poorsolhjouy, "Granular micromechanics model for damage and plasticity of cementitious materials based upon thermomechanics", Mathematics and Mechanics of Solids, vol. 25, no. 10, pp. 1778-1803, 2020.

68. M. Cuomo, "Continuum damage model for strain gradient materials with applications to $1 \mathrm{~d}$ examples”, Continuum Mechanics and Thermodynamics, vol. 31, no. 4, pp. 969-987, 2019.

69. L. Placidi, "A variational approach for a nonlinear 1-dimensional second gradient continuum damage model", Continuum Mechanics and Thermodynamics, vol. 27, no. 4-5, pp. 623-638, 2015.

70. L. Placidi, E. Barchiesi, and A. Misra, "A strain gradient variational approach to damage: a comparison with damage gradient models and numerical results", Mathematics and Mechanics of Complex Systems, vol. 6, no. 2, pp. 77-100, 2018.

71. L. Placidi, A. Misra, and E. Barchiesi, "Two-dimensional strain gradient damage modeling: a variational approach", Zeitschrift für Angewandte Mathematik und Physik, vol. 69(3), no. 56, 2018.

72. L. Placidi, E. Barchiesi, A. Misra, and U. Andreaus, "Variational methods in continuum damage and fracture mechanics", in Encyclopedia of Continuum Mechanics (O. A. Altenbach H, ed.), Encyclopedia of Continuum Mechanics. Springer, Berlin, 2019.

73. L. Placidi, A. Misra, and E. Barchiesi, "Simulation results for damage with evolving microstructure and growing strain gradient moduli", Continuum Mechanics and Thermodynamics, vol. 31, no. 4, pp. 1143-1163, 2019.

74. L. Greco, "An iso-parametric $G^{1}$-conforming finite element for the nonlinear analysis of Kirchhoff rod. Part I: the 2D case", Continuum Mechanics and Thermodynamics, vol. 32, pp. 1473-1496, 2020. 
75. I. Giorgio, "Lattice shells composed of two families of curved Kirchhoff rods: An archetypal example, topology optimization of a cycloidal metamaterial", Continuum Mechanics and Thermodynamics, vol. 33, no. 4, pp. 1063-1082, 2021.

76. A. Ciallella, D. Pasquali, M. Gołaszewski, F. D'Annibale, and I. Giorgio, "A rateindependent internal friction to describe the hysteretic behavior of pantographic structures under cyclic loads", Mechanics Research Communications, vol. 116, 2021, DOI: 10.1016/j.mechrescom.2021.103761.

77. I. Giorgio, "A discrete formulation of Kirchhoff rods in large-motion dynamics", Mathematics and Mechanics of Solids, vol. 25, no. 5, pp. 1081-1100, 2020.

78. I. Giorgio, P. Harrison, F. dell'Isola, J. Alsayednoor, and E. Turco, "Wrinkling in Engineering Fabrics: A Comparison between Two Different Comprehensive Modelling Approaches", Proceedings of the Royal Society of London A, vol. 474(2216), pp. 1-20, 2018.

79. I. Giorgio, V. Varano, F. dell'Isola, and N. L. Rizzi, "Two layers pantographs: A 2D continuum model accounting for the beams' offset and relative rotations as averages in $\mathrm{SO}(3)$ Lie groups", International Journal of Solids and Structures, vol. 216, pp. 43-58, 2021.

80. M. De Angelo, E. Barchiesi, I. Giorgio, and B. E. Abali, "Numerical identification of constitutive parameters in reduced order bi-dimensional models for pantographic structures: Application to out-of-plane buckling", Archive of Applied Mechanics, vol. 89, no. 7, pp. 13331358, 2019.

81. H. Yang, G. Ganzosch, I. Giorgio and B. E. Abali, "Material characterization and computations of a polymeric metamaterial with a pantographic substructure", ZAMP - Zeitschrift für angewandte Mathematik und Physik, vol. 69, no. 4, pp. 1-16, 2018. 